\title{
Embryo and seedling morphology of some Trifolium L. species (Fabaceae)
}

\author{
Faiza A. Shehata*1, Esam M. Aqlan ${ }^{2}$ \\ ${ }^{1}$ Botany \& Microbiology Department, Faculty of Science, Menoufia University, Egypt \\ ${ }^{2}$ Biology Department, Faculty of Science, Ibb University, Ibb, Yemen \\ *Corresponding author: faizashehata@yahoo.com
}

\begin{abstract}
The present study aims to describe and characterize the morphology of the embryo and the seedling and their importance in the taxonomy of the studied Trifolium species. Macro and micromorphological studies were carried on 15 Trifolium species using light microscopy. Qualitative and quantitative characteristics of the embryo, seedling with cotyledonary leaves, seedling with first unifoliate and first trifoliate leaves indicated that cotyledon characters such as cotyledon shape; $\mathrm{L} / \mathrm{W}$ ratio, apex, colour, radicle position; shape, apex; cotyledonary leaves such as hypocotyl colour, blade shape, $L / W$ ratio; first and second leaves (shape, apex base, margin and texture) proved to be useful for identification of different taxa. Numerical analysis is carried out and phenogram illustrating the relationship between the studied taxa was constructed by calculating the average taxonomic distance. Results show a taxonomic significance in the identification and differentiation between the studied taxa. An identification key was prepared based on embryo and seedling morphology.
\end{abstract}

Keywords: embryo, Fabaceae, first \& second foliage morphology, seedling, Trifolium.

\section{Introduction}

The genus Trifolium L. (Fabaceae) includes approximately 250-300 species, mostly annual, biennial and short-lived perennial, distributed throughout the temperate and subtropical regions (Allen \& Allen, 1981; Zohary \& Heller, 1984 and Ellison et al., 2006). The genus is cosmopolitan with species that occur mostly in Northern hemisphere; it includes a large number of food crops and forage plants.

The genus has been divided into eight sections: Chronosemium, Involucrarium, Lotoidea, Mystillus, Paramesus, Tricocephalum, Trifolium and Vesicaria, where Lotoidea is the largest and has served as a source taxon for the evolution of other sections (Zohary \& Heller, 1984).

The seedling stage is arguably the busiest phase in a plant's lifetime. Once germination has occurred, the seedling depends on its own morphological and physiological characteristics to cope with the various factors threatening its survival (Farnsworth, 2008).
Seedling morphological characters are as important, reliable, and conservative characters as that of floral ones, and should be used in the delimitation of different taxonomic groups (Mundhra et al., 2012).

Different workers have also carried out investigations on seedling morphology at the level of genus with reference to their taxonomic importance (Scott \& Smith, 1998).

Morphological studies were carried on seedling giving on different taxonomic groups: Leguminosae (Compton, 1912), Juglandaceae (Conde \& Stone, 1970), Sapotaceae (Bokdam, 1977), Iridaceae (Tillich, 2003) and Malpighicaceae (Barbosa et al., 2014). Seedling morphology has also been utilized in the preparation of seedling flora (Lubbock, 1892, Burger, 1972, Muller, 1978). Seedlings of Dicotyledons are some of the noteworthy contributions in this regard.

Vogel (1980) classified the dicotyledons based on seedling morphology and germination pattern, embryo and seed size. Paria et al. (1991) described seedling 
morphology of 14 species under 13 genera related to four families of Malvales and utilized the data in the construction of artificial keys to the identification of the taxa showing affinities within them. Das (2001) clarified the taxonomic significance of mangrove seedling morphology, depending upon special morphological character like, seedling type, cotyledons differentiation and hypocotyls elongation. Characters of seedlings have taxonomic implications on Bauhinia L. (Bandyopadhyay, 2002). Khalik \& Van der Maesen (2002) used radicle/cotyledons position as a significant character to separate different tribes of Brassicaceae.

Seedlings in Fabaceae were previously studied and provided taxonomic characters that was useful in delimiting different levels of taxonomic groups (Baudet, 1974; Duke \& Polhill, 1981; Ye, 1983; Nozzolillo, 1985; Lima, 1989; Oliveira, 2001 and Rodrigues \& de Azeve do Tozzi, 2007), it was found useful in the tribe Trifoliae in segregating particular species like Medicago (Buendia Lazaro et al., 1966). Sanyall \& Paria (2015) used seedling morphological characters of twenty-five taxa under eighteen genera to determine interrelationships among these taxa and to construct artificial key for identification purpose. Turki et al. (2016) studied 29 Medicago species, used morphological characters of cotyledons, seedlings and first foliage leaves in differentiation between the studied taxa.

Karaismailoglu (2015) treated embryo size as useful taxonomic character of the genus Romulea (Iridaceae) in Turkey. Characters of cotyledons in Fabaceae correlated with taxonomic grouping at several levels within the family (Smith 1983). Based on cotyledons characters; Scott \& Smith (1998) recognized the Acacia subgenera. According to the number of leaf primordial, Nemoto \& Ohashi (1993) recognized two distinct types of plumule in genus Lespedeza. Gavadi and Yamaguchi (2004) used plumule features as a parameter for species identification and resolution of species relationship of genus Cicer.

Numerical analyses as a useful statistical tool play an important role in clarifying the relationships between different taxonomic levels (Van de Wouw et al., 2001; Khalik et al., 2002; Turki et al., 2013 and Kendir et al., 2015).

According to the literature, there is no previous work about embryo and seedling description of the Trifolium species. The present study aims to study the detailed description of the embryo and seedling structure of Trifolium species to evaluate the systematic value of these characters as an aid in recognition of the studied species.

\section{Materials \& Methods}

Seeds of 15 species belonging to Trifolium obtained from the Institute of Plant Genetics and Crop Plant Research (IPK) - Germany (Table 1) and stored at $5 \mathrm{C}^{\circ}$ at refrigerator until their use. Seed soaked (5-10 seeds) in tap water for 2-3 hours, remove seed coat gently, examine by using Stereomicroscope using different magnification.

Seeds planted (7-10 seeds) at about $1 \mathrm{~cm}$ in plastic pots $(10 \mathrm{~cm}$ diameter) filled with soil (sand and peat moss 2:1 respectively), irrigation carried out by tap water.

Seedlings measurements were taken at the first growth stage (cotyledonary leaves stage) and the second growth stage (first unifoliate and trifoliate foliage leaf stage). Description of hypocotyls, petioles and blades followed the terminology used by Hickey (1973), Vogel (1980), Ahammed \& Paria (1996) and Khalik $\&$ Van der Maesen (2002).

For minor details in morphology, the seedlings were examined using stereomicroscope then photographed using Zeiss research microscope using different magnification.

For statistical analysis, characters were encoded according to the multistate method; they were coded as $(0,1)$ for different 
character states; 31 characters included 152 character states were recorded for each taxon. The codes were analyzed with NTsys version 2.1 (Rohlf, 2000) which is specialized in the numerical analysis data. Phenogram illustrating the relationship between the studied taxa were constructed by calculating the average taxonomic distance.

Table 1: List of the studied species of Trifolium $\mathrm{L}$. and the sources of seeds.

\begin{tabular}{|c|c|c|c|}
\hline & Species & Synonyms & $\begin{array}{l}\begin{array}{l}\text { Source of seeds } \\
\text { (IPK) }\end{array} \\
\end{array}$ \\
\hline 1 & Trifolium argutum Banks \& Sol. & $\begin{array}{l}\text { Trifolium xrocphalum Fenzl, } T \text {. } \\
\text { moriferum Boiss. }\end{array}$ & Portugal (PRT) \\
\hline 2 & Trifolium bocconei Savi. & - & Portugal (PRT) \\
\hline 3 & Trifolium campestre Schreb. & $\begin{array}{l}\text { Trifolium agrarium L., T. procumbens L., } \\
\text { T. lagrangei Boiss. }\end{array}$ & Portugal (PRT \\
\hline 4 & Trifolium dubium Sibth. & Trifolium minus Sm. & Portugal (PRT \\
\hline 5 & Trifolium fragiferum $\mathrm{L}$. & $\begin{array}{l}\text { Trifolium congestum Link, T. neglectum } \\
\text { C. Mey. }\end{array}$ & Australia (AUS) \\
\hline 6 & Trifolium glomeratum L. & Amoria glomerata (L.) Sojak & Portugal (PRT) \\
\hline 7 & Trifolium lappaceum L. & Trifolium carteiense Coincy & Portugal (PRT) \\
\hline 8 & Trifolium ligusticum Balb. ex Loisel. & - & Frankreich (FRA) \\
\hline 9 & Trifolium michelianum Savi. & Trifolium macropodum Guss. & Bulgaria (BGR) \\
\hline 10 & Trifolium nigrescens Viv. & Amoria nigrescence (Viv.) Fourr. & Portugal (PRT \\
\hline 11 & Trifolium physodes M. Bieb. & Amoria physcodes (M. Bieb.) Roskov & Portugal (PRT \\
\hline 12 & Trifolium purpureum Loisel. & $\begin{array}{l}\text { Trifolium angustifolium L. } \\
\text { subsp. purpureum (Loisel.) Ponert }\end{array}$ & Lebanon (LBN) \\
\hline 13 & Trifolium repens L. & Amoria repens (L.) C. Presl & Portugal (PRT) \\
\hline 14 & Trifolium squarrosum L. & $\begin{array}{l}\text { Trifolium dipsaceum Thuill., } \\
\text { T. panormitanum C. Presl }\end{array}$ & Italy (IAT) \\
\hline 15 & Trifolium tomentosum L. & Amoria tomentosa (L.) Roskov & Portugal (PRT) \\
\hline
\end{tabular}

\section{Results}

The embryo characters, description of the seedling in the cotyledonary leaves; description of seedling with the first unifoliate leaf and, of the first trifoliate foliage leaf of the fifteen investigated taxa are summarized in tables 1-4 and fig. 1-9.

\section{Description of embryo (Fig. 1-4, Table 2)}

Cotyledons shape were obovate in $T$. glomeratum, T. michelianum, T. nigrescens, $T$. physodes, and $T$. repens, elliptic in $T$. campestre, T. ligusticum and T. tomentosum and oblong in the remaining seven studied species. Cotyledons length $\times$ width were $2.8-3$ $\times 1.2-1.3 \mathrm{~mm}$ in $T$. squarrosum and $0.8-2 \times 0.3-$ $1 \mathrm{~mm}$ in the remaining 14 studied species. Cotyledons were pale brown in T. argutum, $T$. ligusticum, T. michelianum and T. physodes and pale yellow in the remaining 11 species. Radicles were accumbent (i.e. cotyledons having their edges folded against the hypocotyl) in $T$. physodes and incumbent in the remaining 14 studied species. Radicle flattened in T. argutum, semicylindrical in $T$. purpureum and cylindrical in the remaining 13 studied species. Radicle apex were acuminate in $T$. argutum and T. squarrosum, obtuse in $T$. ligusticum, T. nigrescens and T. physodes, rounded in $T$. tomentosum, acute-subacute in $T$. fragiferum and acute in the remaining eight studied species. Radicles length $\times$ width were $0.3-0.5 \times 0.2 \mathrm{~mm}$ in $T$. bocconei, $0.6-1.2 \times 0.2$ $\mathrm{mm}$ in $T$. campestre, $T$. dubium, $T$. glomeratum, T. lappaceum, $T$. ligusticum, $T$. nigrescens, and $T$. purpureum, 1.3-1.7 $\times 0.2$ $\mathrm{mm}$ in $T$. fragiferum, $T$. michelianum, $T$. physodes, T. repens and T. tomentosum, 1.9-2 $\times 0.2 \mathrm{~mm}$ in T. argutum and up to $2.3-2.5 \times 0.2$ $\mathrm{mm}$ in T. squarrosum. Mucilage attached to embryo in $T$. bocconei, $T$. fragiferum, $T$. lappaceum, T. nigrescens, $T$. physodes, $T$. purpureum, T. repens, T. squarrosum and $T$. tomentosum and not attached in the remaining six studied species. 

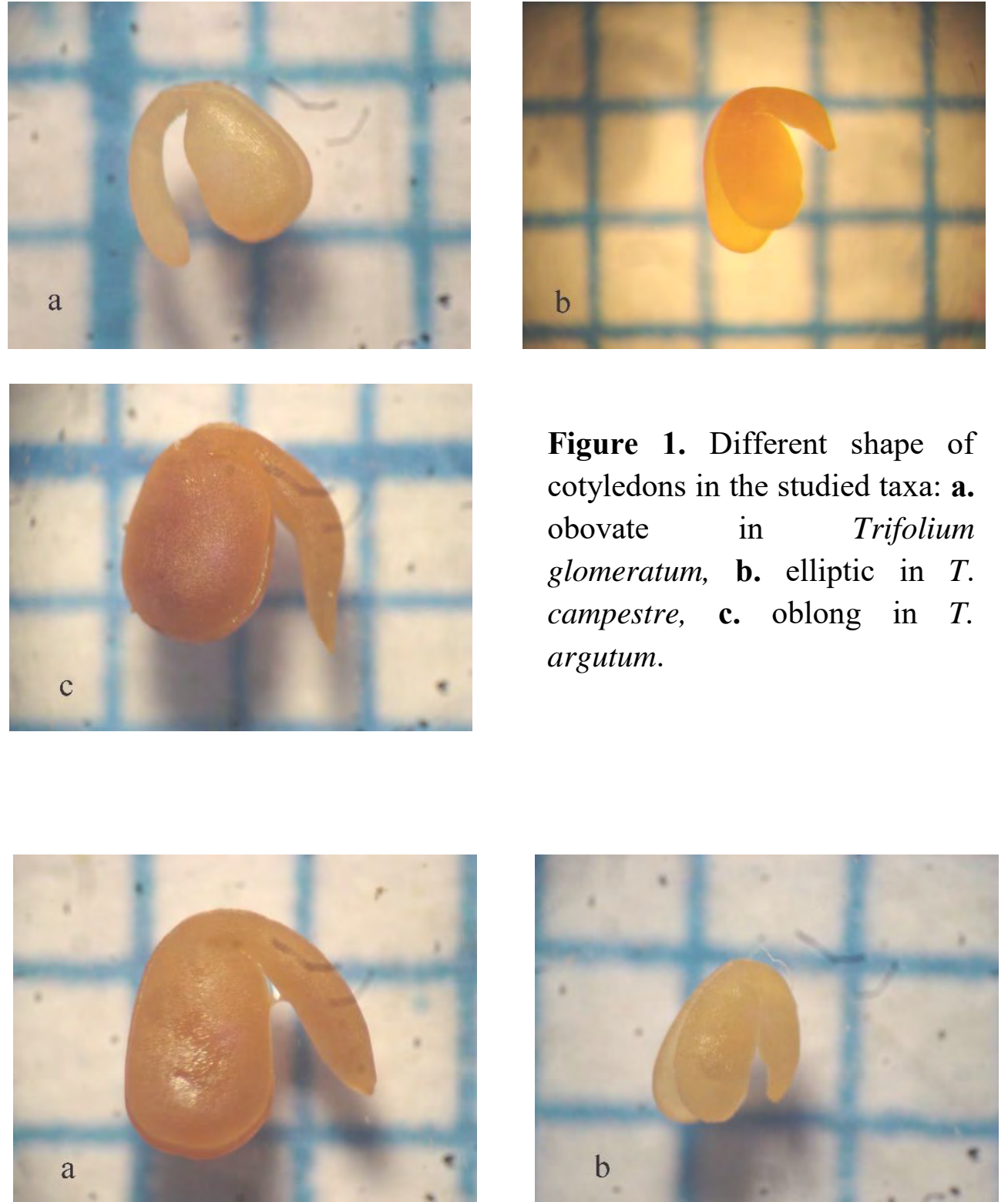

Figure 2. Cotyledons colour: a. pale brown in Trifolium argutum, b. pale yellow in $T$. bocconei
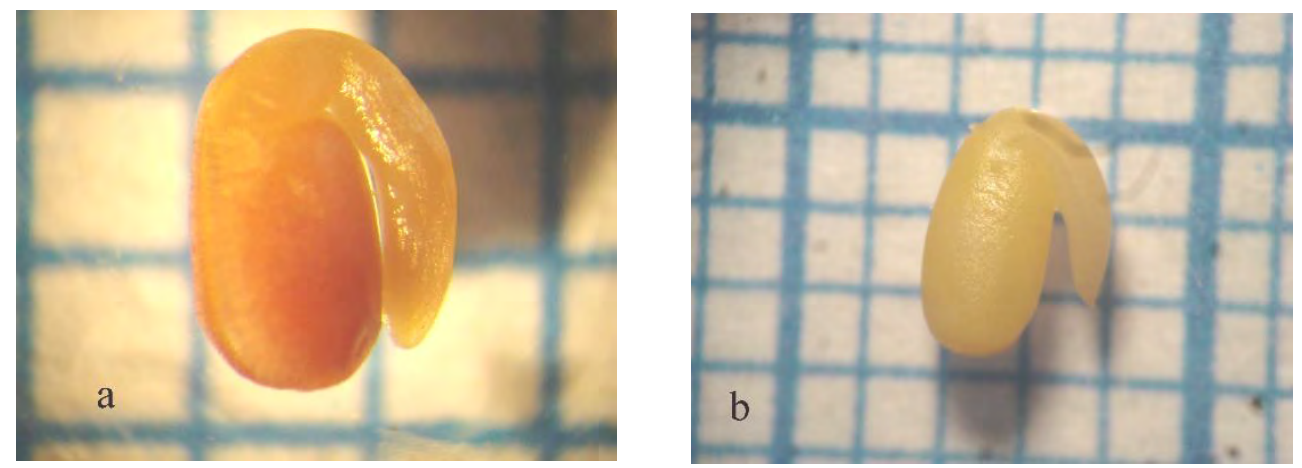

Figure 3. a. accumbent radicle in Trifolium physodes, b. incumbent radicle in $T$. argutum. 

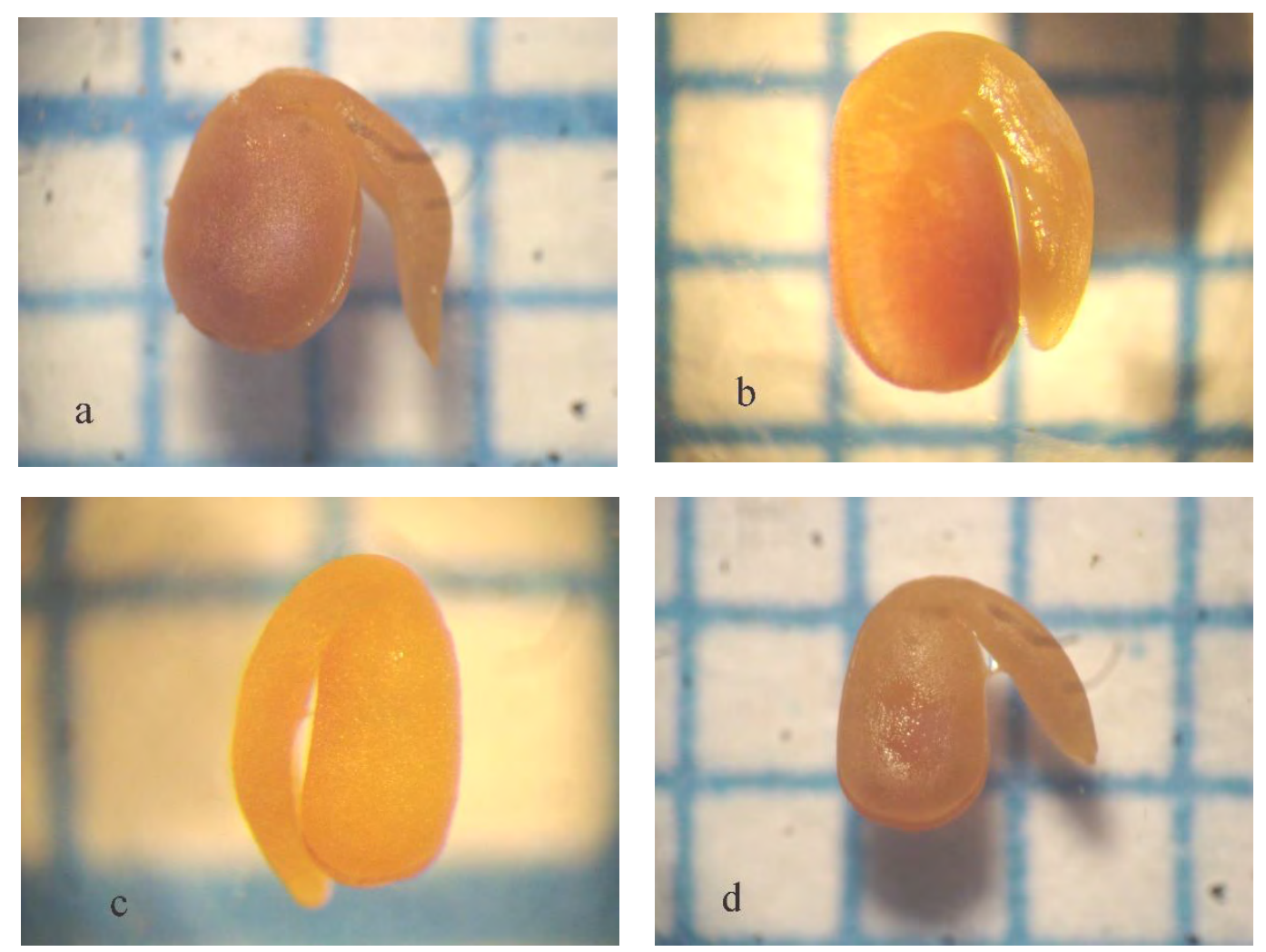

Figure 4. Radicle apex: a. acuminate in Trifolium argutum, b. obtuse in $T$. ligusticum, c. rounded in T. tomentosum, d. acute in T. bocconei.

\section{Description of the seedling in the cotyledonary leaves stage (Fig. 5, Table 3)}

Hypocotyl colour yellowish green in $T$. repens, green in $T$. fragiferum, $T$. physodes and T. squarrosum, reddish green in T. lappaceum, whitish green in $T$. argutum and pale green in the remaining nine studied species. Hypocotyl length up to 27-28 $\mathrm{mm}$ in T. purpureum, 17-19 $\mathrm{mm}$ in T. dubium and T. physodes, $14-15 \mathrm{~mm}$ in $T$. argutum, $10-12 \mathrm{~mm}$ in $T$. campestre and T. ligusticum,7-10 $\mathrm{mm}$ in $T$. fragiferum, $T$. lappaceum T. michelianum, and $T$. squarrosum, and $1-5 \mathrm{~mm}$ in the remaining five species. Length of the cotyledonary leaf petiole were $4.5-7 \mathrm{~mm}$ in $T$. argutum, $T$. fragiferum, T. michelianum, and T. physodes, and were $2-3 \mathrm{~mm}$ in $T$. glomeratum, $T$. lappaceum, T. purpureum, T. squarrosum and T. tomentosum and up to $2 \mathrm{~mm}$ in the remaining 6 studied species. Blade shape obovate in $T$. glomeratum and T. squarrosum, oblong in the remaining 13 studied species. Blade apex obtuse in T. ligusticum and $T$. repens while rounded in the remaining 13 studied species. Blade base cuneate in $T$. glomeratum and $T$. tomentosum while rounded in the remaining 13 studied species. Blade length $\times$ width; 7-8 $\times 2-3 \mathrm{~mm}$ in T. squarrosum, 4-6 $\times 2-3.5 \mathrm{~mm}$ in $T$. argutum, T. lappaceum, $T$. ligusticum, $T$. michelianum, and $T$. physodes, $1.2-1.5 \times 0.8-1 \mathrm{~mm}$ in $T$. purpureum and up to $4 \times 1.3-2 \mathrm{~mm}$ in the remaining eight studied species. 
Table 2. Characters of embryo structure of 15 studied Trifolium species

\begin{tabular}{|c|c|c|c|c|c|c|c|c|c|}
\hline \multirow[b]{2}{*}{ Species } & \multicolumn{4}{|c|}{ Cotyledon } & \multicolumn{4}{|c|}{ Radicle } & \multirow{2}{*}{$\begin{array}{l}\text { Attachment } \\
\text { of } \\
\text { mucilage } \\
\text { to embryo }\end{array}$} \\
\hline & Shape & $\begin{array}{l}\mathrm{L} \times \mathrm{W} \\
(\mathrm{mm})\end{array}$ & $\begin{array}{l}\mathrm{L} / \mathrm{W} \\
\text { ratio }\end{array}$ & Colour & Position & Shape & Apex & $\begin{array}{l}\mathrm{L} \times \mathrm{W} \\
(\mathrm{mm})\end{array}$ & \\
\hline T. argutum & oblong & $\begin{array}{r}1.6-1.8 \\
\times 0.9-1\end{array}$ & 1.7 & $\begin{array}{l}\text { pale } \\
\text { brown }\end{array}$ & incumbent & flattened & acuminate & $\begin{array}{l}1.9-2 \times \\
0.2\end{array}$ & $\begin{array}{l}\text { not } \\
\text { attached }\end{array}$ \\
\hline T. bocconei & oblong & $\begin{array}{l}0.8-1 \times \\
0.3-0.4\end{array}$ & 2.5 & $\begin{array}{l}\text { pale } \\
\text { yellow }\end{array}$ & incumbent & cylindrical & acute & $\begin{array}{l}0.3-0.5 \\
\times 0.2\end{array}$ & attached \\
\hline T. campestre & elliptic & $\begin{array}{l}1.3-1.5 \\
\times 0.5- \\
0.6\end{array}$ & 2.5 & $\begin{array}{l}\text { pale } \\
\text { yellow }\end{array}$ & incumbent & cylindrical & acute & $\begin{array}{l}0.8-0.9 \\
\times 0.2\end{array}$ & $\begin{array}{l}\text { not } \\
\text { attached }\end{array}$ \\
\hline T. dubium & oblong & $\begin{array}{l}1.2-1.5 \\
\times 0.5\end{array}$ & 2.7 & $\begin{array}{l}\text { pale } \\
\text { yellow }\end{array}$ & incumbent & cylindrical & acute & $\begin{array}{l}0.8-0.9 \\
\times 0.2\end{array}$ & $\begin{array}{l}\text { not } \\
\text { attached }\end{array}$ \\
\hline T. fragiferum & oblong & $\begin{array}{l}1.5-2 \times \\
0.8-1\end{array}$ & 1.9 & $\begin{array}{l}\text { pale } \\
\text { yellow }\end{array}$ & incumbent & cylindrical & $\begin{array}{l}\text { acute- } \\
\text { subacute }\end{array}$ & $\begin{array}{l}1.3-1.5 \\
\times 0.2\end{array}$ & attached \\
\hline T. glomeratum & obovate & $\begin{array}{l}1.3-.5 \times \\
0.6\end{array}$ & 2.3 & $\begin{array}{l}\text { pale } \\
\text { yellow }\end{array}$ & incumbent & cylindrical & acute & $\begin{array}{l}0.7-0.9 \\
\times 0.2\end{array}$ & $\begin{array}{l}\text { not } \\
\text { attached }\end{array}$ \\
\hline T. lappaceum & oblong & $\begin{array}{l}1.8-2 \times \\
0.8-1\end{array}$ & 2.1 & $\begin{array}{l}\text { pale } \\
\text { yellow }\end{array}$ & incumbent & cylindrical & acute & $\begin{array}{l}1-1.2 \times \\
0.2\end{array}$ & attached \\
\hline T. ligusticum & elliptic & $\begin{array}{l}1.2-1.4 \\
\times 0.8\end{array}$ & 1.6 & $\begin{array}{l}\text { pale } \\
\text { brown }\end{array}$ & incumbent & cylindrical & obtuse & $\begin{array}{l}0.6-0.7 \\
\times 0.2\end{array}$ & $\begin{array}{l}\text { not } \\
\text { attached }\end{array}$ \\
\hline T. michelianum & obovate & $\begin{array}{l}1.7-1.9 \\
\times 0.4\end{array}$ & 4.5 & $\begin{array}{l}\text { pale } \\
\text { brown }\end{array}$ & incumbent & cylindrical & acute & $\begin{array}{l}1.4-1.5 \\
\times 0.2\end{array}$ & $\begin{array}{l}\text { not } \\
\text { attached }\end{array}$ \\
\hline T. nigrescens & obovate & $\begin{array}{l}0.8-1 \times \\
0.3-0.4\end{array}$ & 2.5 & $\begin{array}{l}\text { pale } \\
\text { yellow }\end{array}$ & incumbent & cylindrical & obtuse & $\begin{array}{l}0.7-0.8 \\
\times 0.2\end{array}$ & attached \\
\hline T. physodes & obovate & $\begin{array}{l}1.8-2 \\
\times 1\end{array}$ & 1.9 & $\begin{array}{l}\text { pale } \\
\text { brown }\end{array}$ & accumbent & cylindrical & obtuse & $\begin{array}{l}1.4-1.6 \\
\times 0.2\end{array}$ & attached \\
\hline T. purpureum & oblong & $\begin{array}{l}1.8-2 \times \\
0.9-1\end{array}$ & 2 & $\begin{array}{l}\text { pale } \\
\text { yellow }\end{array}$ & incumbent & $\begin{array}{l}\text { semi- } \\
\text { cylindrical }\end{array}$ & acute & $\begin{array}{l}1-1.2 \times \\
0.2\end{array}$ & attached \\
\hline T. repens & obovate & $\begin{array}{l}1.2-1.4 \\
\times 0.8\end{array}$ & 1.6 & $\begin{array}{l}\text { pale } \\
\text { yellow }\end{array}$ & incumbent & cylindrical & acute & $\begin{array}{l}1.4-1.6 \\
\times 0.2\end{array}$ & attached \\
\hline T. squarrosum & oblong & $\begin{array}{l}2.8-3 \times \\
1.2-1.3\end{array}$ & 2.3 & $\begin{array}{l}\text { pale } \\
\text { yellow }\end{array}$ & incumbent & cylindrical & acuminate & $\begin{array}{l}2.3-2.5 \\
\times 0.2\end{array}$ & attached \\
\hline T. tomentosum & elliptic & $\begin{array}{l}1.2-1.5 \\
\times 0.8\end{array}$ & 1.6 & $\begin{array}{l}\text { pale } \\
\text { yellow }\end{array}$ & incumbent & cylindrical & rounded & $\begin{array}{l}1.5-1.7 \\
\times 0.2\end{array}$ & attached \\
\hline
\end{tabular}
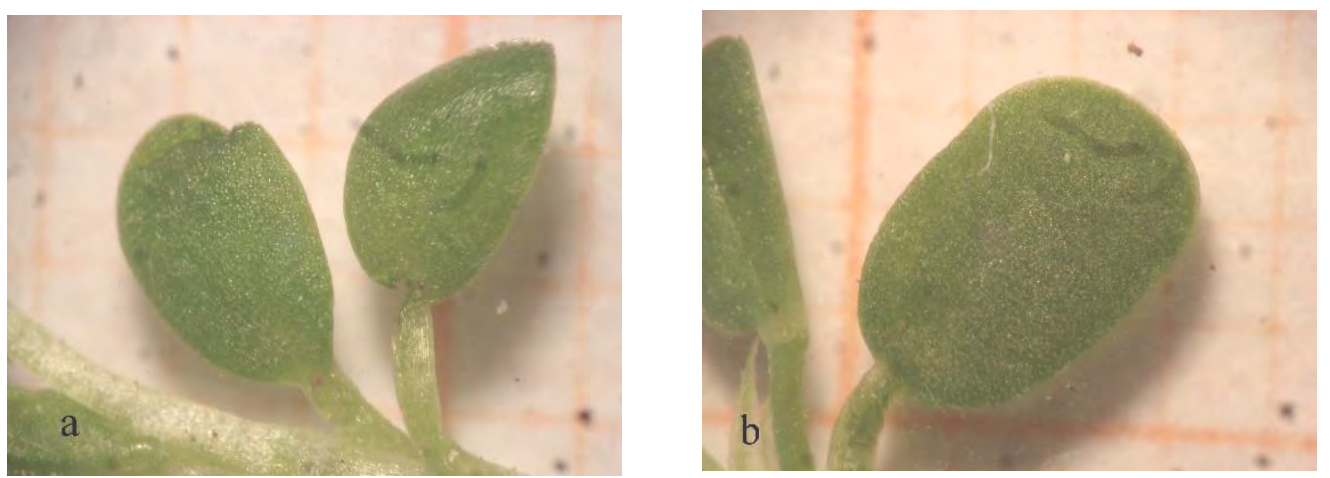

Figure 5. Different shapes of cotyledonary leaf: a. obovate in Trifolium glomeratum, b. oblong in T. campestre. 
Table 3. Description of the seedling in the cotyledonary leaves stage.

\begin{tabular}{|c|c|c|c|c|c|c|c|c|}
\hline \multirow{2}{*}{ Species } & \multicolumn{2}{|c|}{ Hypocotyl } & \multirow{2}{*}{$\begin{array}{l}\text { Petiole } \\
\text { length } \\
(\mathrm{mm})\end{array}$} & \multirow[b]{2}{*}{ Shape } & \multicolumn{3}{|c|}{ Blade } & \multirow[b]{2}{*}{$\begin{array}{l}\mathrm{L} / \mathrm{W} \\
\text { ratio }\end{array}$} \\
\hline & Colour & $\begin{array}{l}\text { Length } \\
(\mathrm{mm})\end{array}$ & & & Apex & Base & $\mathrm{L} \times \mathrm{W}(\mathrm{mm})$ & \\
\hline T. argutum & $\begin{array}{l}\text { whitish } \\
\text { green }\end{array}$ & $14-15$ & $6-7$ & oblong & round & round & $5-6 \times 2.5-3$ & 2 \\
\hline T. bocconei & pale green & $4-5$ & $1-1.5$ & oblong & round & round & $2.5-3 \times 1.5-2$ & 1.5 \\
\hline T. campestre & pale green & $11-12$ & $1-1.5$ & oblong & round & round & $2.8-3 \times 1.8-2$ & 1.5 \\
\hline T. dubium & pale green & $17-18$ & $1-1.5$ & oblong & round & round & $2.8-3 \times 1.3-1.5$ & 2.1 \\
\hline T. fragiferum & green & $9-10$ & $5.5-6.5$ & oblong & round & round & $3-4 \times 2-2.5$ & 1.5 \\
\hline T. glomeratum & pale green & $2-3$ & $2.5-2.8$ & obovate & round & cuneate & $3.5-4 \times 1.8-2$ & 1.9 \\
\hline T. lappaceum & $\begin{array}{l}\text { reddish } \\
\text { green }\end{array}$ & $9-10$ & $2-2.5$ & oblong & round & round & $4-5 \times 2-3$ & 1.6 \\
\hline T. ligusticum & pale green & $11-12$ & $1.5-2$ & oblong & obtuse & round & $4-5 \times 2-3$ & 1.8 \\
\hline T. michelianum & pale green & $7-8$ & $6-6.5$ & oblong & round & round & $4-5 \times 2.5-3$ & 1.6 \\
\hline T. nigrescens & pale green & $4-5$ & $1.5-2$ & oblong & round & round & $2.3-2.5 \times 1.5-2$ & 1.3 \\
\hline T. physodes & green & $18-19$ & $4.8-5$ & oblong & round & round & $5-6 \times 3.2-3.5$ & 1.6 \\
\hline T. purpureum & pale green & $27-28$ & $2-2.5$ & oblong & round & round & $1.2-1.5 \times 0.8-1$ & 1.5 \\
\hline T. repens & $\begin{array}{l}\text { yellowish } \\
\text { green }\end{array}$ & $1-1.2$ & $1.3-1.5$ & oblong & obtuse & round & $2.3-2.5 \times 1-1.5$ & 1.9 \\
\hline T. squarrosum & green & $9-10$ & $2-2.5$ & obovate & round & round & $7-8 \times 2-3$ & 3 \\
\hline T. tomentosum & pale green & $2-2.5$ & $2-2.5$ & oblong & round & cuneate & $3.5-4 \times 1.5-2$ & 2.1 \\
\hline
\end{tabular}

\section{Description of seedling with the first} unifoliate leaf stage (Fig. 6-7, Table 4)

Petiole length up to $39-41 \mathrm{~mm}$ in $T$. purpureum, $28-31 \mathrm{~mm}$ in $T$. argutum and $T$. physodes, $23-27 \mathrm{~mm}$ in $T$. michelianum and $T$. tomentosum, $17-20 \mathrm{~mm}$ in $T$. fragiferum and $T$. ligusticum, 12-15 $\mathrm{mm}$ in $T$. glomeratum and $T$. nigrescens and up to $10 \mathrm{~mm}$ in the remaining six studied species.

Petiole surface pubescent in T. fragiferum and T. ligusticum, pilose in T. bocconei, $T$. lappaceum, T. purpureum and T. squarrosum and glabrous in the remaining nine studied species. Blade shape cordate in $T$. repens, obcordate in $T$. michelianum, oblong in $T$. argutum, oblate in $T$. dubium, T. lappaceum and $T$. ligusticum, elliptic in T. purpureum, square in T. bocconei, ovate in T. physodes and $T$. tomentosum, depressed ovate in $T$. campestre and broadly ovate in $T$. fragiferum, T. glomeratum and T. nigrescens. Blade length $\times$ width; $11-12 \times 5-6 \mathrm{~mm}$ in T. purpureum, 6$7 \times 5-6 \mathrm{~mm}$ in $T$. dubium, $T$. fragiferum, $T$. physodes and $T$. squarrosum, 4-5 × 4-6 $\mathrm{mm}$ in T. campestre, $T$. ligusticum and $T$. tomentosum, up to $2 \times 2 \mathrm{~mm}$ in $T$. repens, 3 $3.5 \times 3.5-4 \mathrm{~mm}$ in T. michelianum, 4-4.5 $\times 3$ 3.5 in $T$. argutum, $2.5-3 \times 2.8-3.5 \mathrm{~mm}$ in $T$. lappaceum, $3.5-4 \times 3.5-4.5 \mathrm{~mm}$ in $T$. bocconei and $T$. glomeratum, and 2.5-3 $\times 3-3.5 \mathrm{~mm}$ in $T$. nigrescens. Blade apex acute in $T$. argutum and $T$. tomentosum, emarginate-apiculate in $T$. glomeratum, rounded in $T$. lappaceum and truncate in $T$. michelianum and emarginate in the remaining ten studied species. Blade base rounded in $T$. argutum and $T$. lappaceum, reniform in $T$. nigrescens, cuneate in $T$. purpureum and $T$. squarrosum, and truncate in the remaining ten studied species. Blade margin ciliate in $T$. argutum, dentate in $T$. fragiferum, T. glomeratum, T. nigrescens, $T$. physodes, $T$. repens and $T$. tomentosum, serrate in T. michelianum and entire in the remaining seven studied species. Blade surface pilose in T. bocconei, T. lappaceum, T. purpureum and $T$. squarrosum, pubescent in $T$. ligusticum, subglabrous in $T$. fragiferum and glabrous in the remaining nine studied species. 

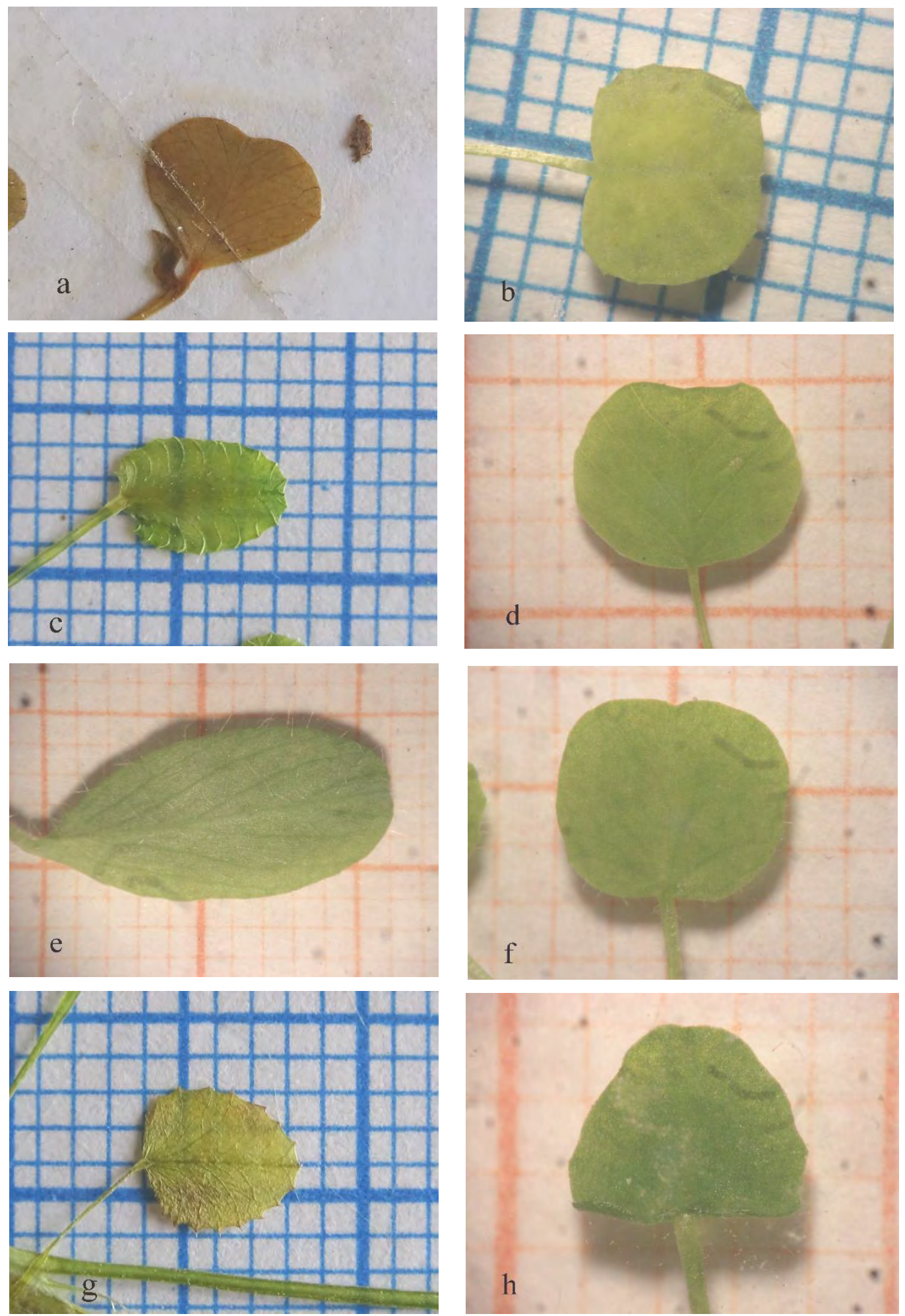

Figure 6. The first unifoliate leaf shape: a. obcordate in Trifoium michelianum, b. depressed ovate in T. campestre, c. oblong in T. argutum, d. oblate in T. lappaceum, e. elliptic in T. purpureum, f. square in T. bocconei, g. ovate in $T$. physodes, h. broadly ovate in $T$. fragiferum. 
Faiza Shehata \& Esam Aqlan
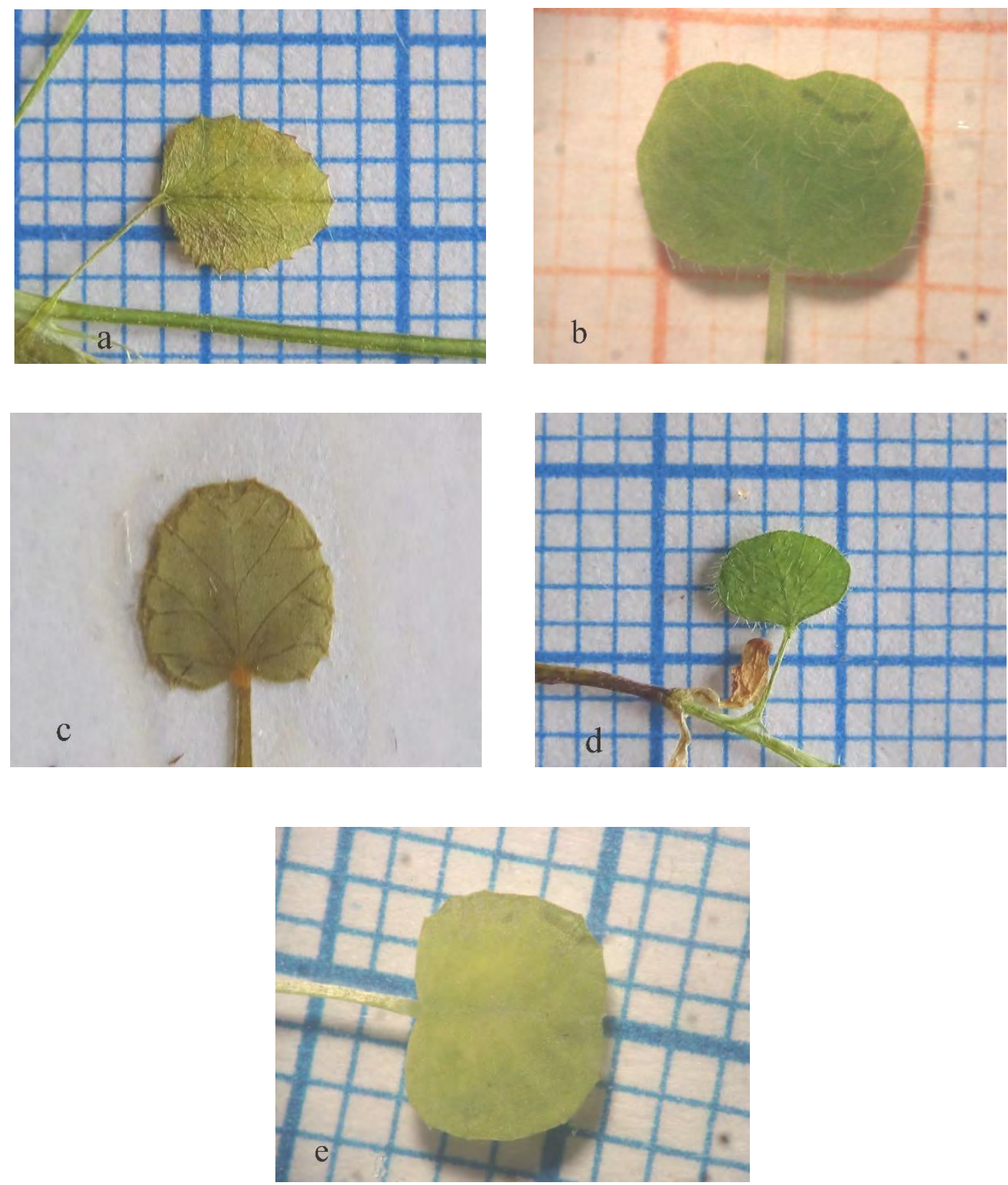

Figure 7. The first unifoliate leaf apex: a. acute in Trifolium argutum, b. emarginate in T. bocconei, c. emarginate-apiculate in T. glomeratum, d. rounded in T. lappaceum, e. truncate in T. michelianum. 
Embryo and seedling morphology of some Trifolium L. species

Table 4. Description of seedling with the first unifoliate leaf stage

\begin{tabular}{|c|c|c|c|c|c|c|c|c|}
\hline \multirow[b]{2}{*}{ Species } & \multirow{2}{*}{$\begin{array}{l}\text { Petiole } \\
\text { Length } \\
(\mathrm{mm})\end{array}$} & \multicolumn{7}{|c|}{ Blade } \\
\hline & & Shape & $\mathrm{L} \times \mathrm{W}(\mathrm{mm})$ & $\begin{array}{l}\mathrm{L} / \mathrm{W} \\
\text { ratio }\end{array}$ & Apex & Base & Margin & Surface \\
\hline T. argutum & $30-31$ & oblong & $4-4.5 \times 3-3.5$ & 1.3 & Acute & rounded & ciliate & glabrous \\
\hline T. bocconei & $7-8$ & square & $3.5-4 \times 3.5-4$ & 1 & emarginate & truncate & entire & pilose \\
\hline T. campestre & $5-6$ & $\begin{array}{l}\text { depressed } \\
\text { ovate }\end{array}$ & $4-5 \times 5-6$ & 0.8 & emarginate & truncate & entire & glabrous \\
\hline T. dubium & $9-10$ & oblate & $4-4.5 \times 5-5.5$ & 0.8 & emarginate & truncate & entire & glabrous \\
\hline T. fragiferum & $17-18$ & $\begin{array}{l}\text { broadly } \\
\text { ovate }\end{array}$ & $4-4.5 \times 4.3-4.8$ & 0.9 & emarginate & truncate & dentate & subglabrous \\
\hline T. glomeratum & $13-15$ & $\begin{array}{l}\text { broadly } \\
\text { ovate }\end{array}$ & $3.5-4 \times 4-4.5$ & 1.1 & $\begin{array}{l}\text { emarginate } \\
\text { apiculate }\end{array}$ & truncate & dentate & glabrous \\
\hline T. lappaceum & $3-4$ & oblate & $2.5-3 \times 2.8-3.5$ & 0.8 & rounded & rounded & entire & pilose \\
\hline T. ligusticum & $18-20$ & oblate & $4-5 \times 5-6$ & 0.8 & emarginate & truncate & entire & pubescent \\
\hline $\begin{array}{l}T . \\
\text { michelianum }\end{array}$ & $23-25$ & obcordate & $3-3.5 \times 3.5-4$ & 0.8 & truncate & truncate & serrate & glabrous \\
\hline T. nigrescens & $12-13$ & $\begin{array}{l}\text { broadly } \\
\text { ovate }\end{array}$ & $2.5-3 \times 3-3.5$ & 0.8 & emarginate & reniform & dentate & glabrous \\
\hline T. physodes & $28-29$ & ovate & $6-7 \times 5-6$ & 1.1 & emarginate & truncate & $\begin{array}{l}\text { dentate } \\
\text { (at upper } \\
\text { third) }\end{array}$ & glabrous \\
\hline T. purpureum & $39-41$ & elliptic & $11-12 \times 5-6$ & 2.1 & emarginate & cuneate & entire & pilose \\
\hline T. repens & $7-8$ & cordate & $1.8-2 \times 1.8-2$ & 1 & emarginate & truncate & $\begin{array}{l}\text { dentate (at } \\
\text { upper half) }\end{array}$ & glabrous \\
\hline T. squarrosum & $5-6$ & obovate & $6-7 \times 5-6$ & 1.1 & emarginate & cuneate & entire & pilose \\
\hline T. tomentosum & $25-27$ & ovate & $4-5 \times 4-5$ & 1 & Acute & truncate & dentate & glabrous \\
\hline
\end{tabular}

\section{Description of the first trifoliate foliage leaf stage (Fig. 8-9, Table 5)}

Leaf petiole length show wide variation; 4-42 $\mathrm{mm}$ in the studied species. Petiole surface pilose in T. bocconei, T. purpureum, and $T$. squarrosum, pubescent in $T$. ligusticum, subglaborus in T. lappaceum, glabrous in the remaining ten studied species. Leaflet shape circular in $T$. nigrescens, elliptic in $T$. fragiferum, $T$. physodes and T. tomentosum, obovate in $T$. purpureum, Semicircular in $T$. argutum, oblong in $T$. squarrosum, obcordate in the remaining eight studied species. Blade length $\times$ width shows differentiation between the studied 15 species listed in the table. Blade apex acuminate in $T$. tomentosum, acute in $T$.

argutum, obtuse in T. purpureum, emarginate in the remaining 12 studied species. Leaf base rounded in T. michelianum, T. nigrescens and T. physodes, truncated in T. dubium, cuneate in the remaining 11 studied species. Leaf margin ciliate in $T$. argutum, serrate in T. michelianum and $T$. repens, dentate in $T$. fragiferum, $T$. glomeratum, T. physodes and T. tomentosum, entire in the remaining eight studied species. Blade surface pubescent in T. ligusticum, pilose in $T$. bocconei, $T$. lappaceum, $T$. purpureum and T. squarrosum, and glabrous in the remaining ten studied species. 

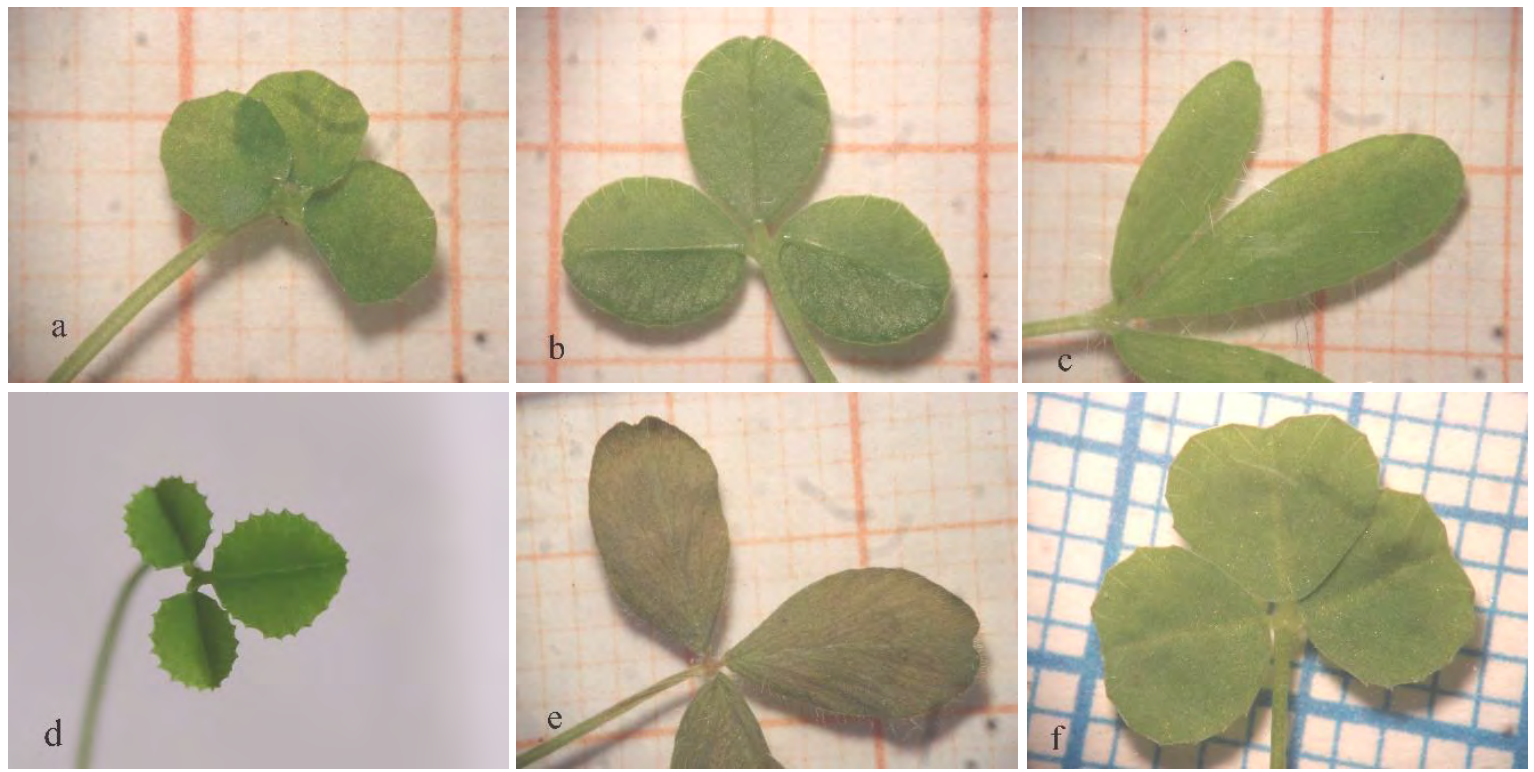

Figure 8. The first trifoliate leaflet shape: a. circular in Trifolium nigrescens, b. elliptic in T. fragiferum, c. obovate in T. purpureum, d. semicircular in T. argutum, e. oblong in T. squarrosum, f. obcordate in T. bocconei.
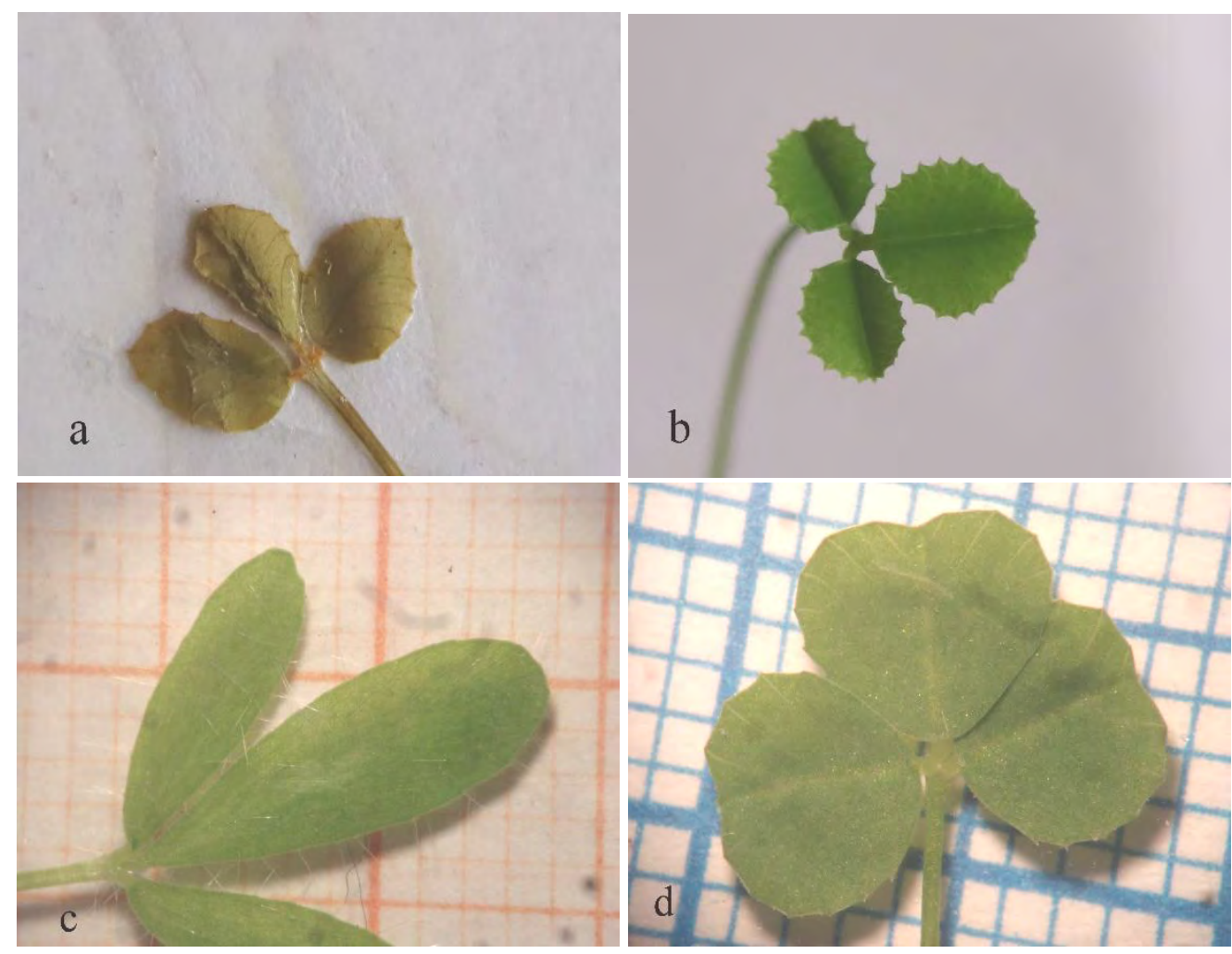

Figure 9. The first trifoliate leaflet apex: a. acuminate in T. tomentosum, b. acute in $T$. argutum, c. obtuse in T. purpureum, d. emarginate in T. bocconei. 
Table 5. Description of seedlings in the first trifoliate foliage leaf stage

\begin{tabular}{|c|c|c|c|c|c|c|c|c|c|}
\hline Species & $\begin{array}{l}\text { Petiole } \\
\text { Length } \\
\mathrm{mm}\end{array}$ & Surface & $\begin{array}{l}\text { Blade } \\
\text { Shape } \\
\text { of leaflet }\end{array}$ & $\begin{array}{c}\mathrm{L} \times \mathrm{W} \\
\mathrm{mm}\end{array}$ & $\begin{array}{l}\mathrm{L} / \mathrm{W} \\
\text { ratio }\end{array}$ & Apex & Base & Margin & Surface \\
\hline T. argutum & $28-30$ & glabrous & semicircular & $\begin{array}{l}4-4.5 \\
\times 3-3.5\end{array}$ & 1.3 & acute & cuneate & ciliate & glabrous \\
\hline T. bocconei & $11-12$ & pilose & obcordate & $\begin{array}{l}3-3.5 \\
\times 3-3.5\end{array}$ & 1 & emarginate & cuneate & entire & pilose \\
\hline T. campestre & $5-6$ & glabrous & obcordate & $\begin{array}{l}3-3.5 \\
\times 3-3.5\end{array}$ & 1 & emarginate & cuneate & entire & glabrous \\
\hline T. dubium & $11-12$ & glabrous & obcordate & $\begin{array}{l}3-3.5 \\
\times 2.5-3\end{array}$ & 1.2 & emarginate & truncate & entire & glabrous \\
\hline T. fragiferum & $25-26$ & glabrous & elliptic & $\begin{array}{l}4-4.5 \\
\times 3.5-4\end{array}$ & 1.3 & emarginate & cuneate & dentate & glabrous \\
\hline T. glomeratum & $20-21$ & glabrous & obcordate & $\begin{array}{l}3-3.5 \\
\times 3-3.5\end{array}$ & 1 & emarginate & cuneate & dentate & glabrous \\
\hline T. lappaceum & $4-5$ & subglaborus & obcordate & $\begin{array}{l}2.8-3 \\
\times 2.8-3\end{array}$ & 1 & emarginate & cuneate & entire & pilose \\
\hline T. ligusticum & $32-33$ & pubescent & obcordate & $\begin{array}{l}3.5-4 \\
\times 4.5-5\end{array}$ & 0.7 & emarginate & cuneate & entire & pubescent \\
\hline T. physodes & $40-42$ & glabrous & obcordate & $\begin{array}{l}4-4.5 \\
\times 4-5\end{array}$ & 0.9 & emarginate & round & serrate & glabrous \\
\hline T. nigrescens & $15-16$ & gabrous & circular & $\begin{array}{l}2-2.5 \\
\times 2-2.5\end{array}$ & 1 & emarginate & round & entire & glabrous \\
\hline T. michelianum & $20-22$ & glabrous & elliptic & $\begin{array}{l}3-4 \times 2- \\
3\end{array}$ & 1.4 & emarginate & round & dentate & glabrous \\
\hline T. purpureum & $40-41$ & pilose & obovate & $\begin{array}{l}10-11 \\
\times 3-3.5\end{array}$ & 3.2 & obtuse & cuneate & entire & pilose \\
\hline T. repens & $11-12$ & glabrous & obcordate & $\begin{array}{l}5-5.5 \\
\times 4-4.5\end{array}$ & 1.2 & emarginate & cuneate & serrate & glabrous \\
\hline T. squarrosum & $20-21$ & pilose & oblong & $\begin{array}{l}7-7.5 \\
\times 3.5-4\end{array}$ & 1.9 & emarginate & cuneate & entire & pilose \\
\hline T. tomentosum & $28-30$ & glabrous & elliptic & $\begin{array}{l}3.5-4 \\
\times 2.5-3 \\
\end{array}$ & 1.3 & acuminate & cuneate & dentate & glabrous \\
\hline
\end{tabular}

\section{Numerical analysis}

The dendrogram produced from using distance measure (Fig. 10) showed that on the bases of total characters (Appendix 1), Trifolium species was split off at distance level 1.67 into two series; the first series represented by $T$. argutum which characterized by flattened radicle shape, whitish green hypocotyl colour, oblong unifoliate leaf, with ciliate margin, second leaf with acute apex and ciliate margin.
The second series include the remaining 14 studied species and characterized by cylindrical, semi-cylindrical radicle shape, pale green, reddish green, yellowish green hypocotyl colour; unifoliate leaf shape square, depressed ovate, oblate, broadly ovate, obcordate, ovate, elliptic, cordate, obovate with entire, dentate, serrate margin, the second leaf with emarginated, obtuse, acuminate margin, and entire, serrate margin. 


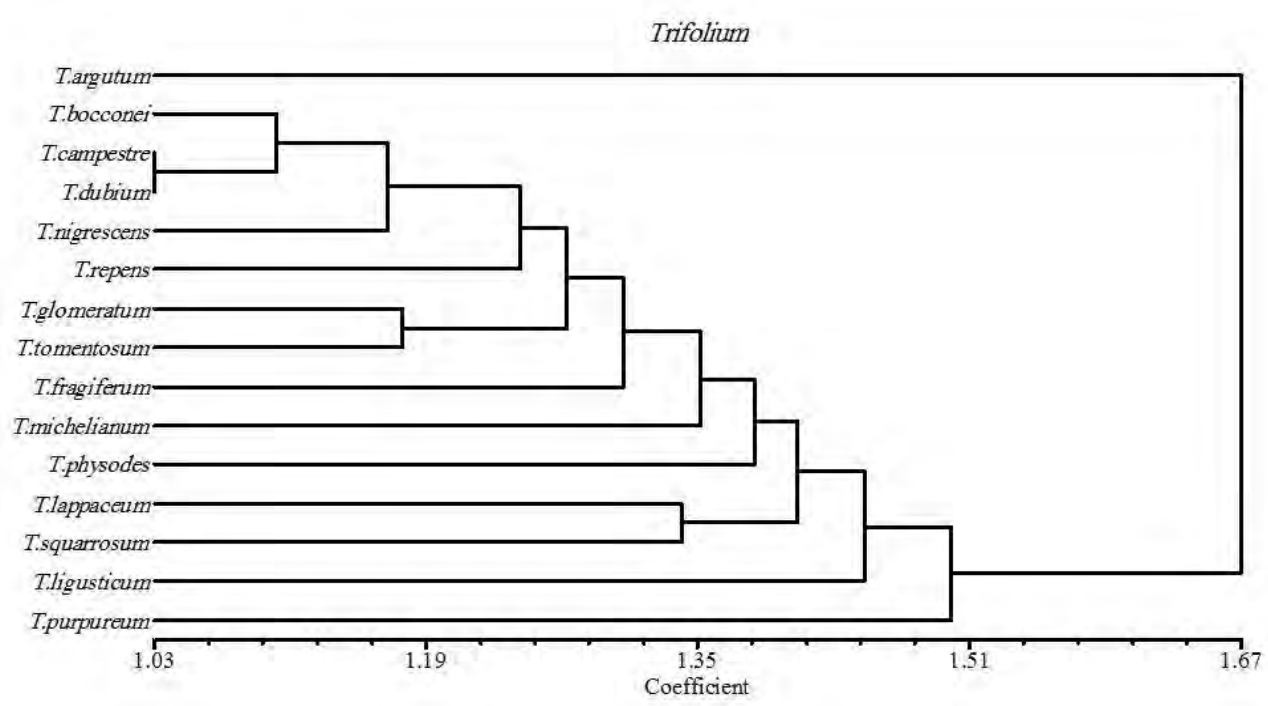

Figure 10. Dendrogram illustrating the relationships between the studied 15 Trifolium species on the bases of the morphological characters of the embryo and the seedlings cotyledonary leaves and the first unifoliate and trifoliate leaf stages.

The second series spilt off at distance level 1.49 into two subseries, the first represented by $T$. purperum and characterized by semicylindrical radicle shape, hypocotyl length $27-$ $28 \mathrm{~mm}$, petiole length of unifoliate leaf $39-41$ $\mathrm{mm}$, elliptic unifoliate leaf shape and $\mathrm{L} / \mathrm{W}$ ratio 2.1 , the second leaf $\mathrm{L} / \mathrm{W}$ ratio 3.2 , with obtuse apex.

The second subseries characterized by cylindrical radicle shape, hypocotyl length up to $19 \mathrm{~mm}$, petiole length of unifoliate leaf up to $29 \mathrm{~mm}$, square, depressed, ovate, oblate, broadly ovate obcordate, ovate, cordate, obovate unifoliate leaf shape and $\mathrm{L} / \mathrm{W}$ ratio 1.1 , second leaf $\mathrm{L} / \mathrm{W}$ ratio up to 1.9 , with emarginated, acuminate apex.

The second subseries split off at distance level 1.45 into two clusters, the first cluster represented by $T$. ligusticum and characterized by pubescent unifoliate leaf surface and petiole and surface of second leaf, $\mathrm{L} / \mathrm{W}$ ratio of the second leaf up to 0.7 , the second cluster represented by the remaining studied species and characterized by glabrous, subglaborus, pilose unifoliate leaf surface, glabrous, subglabrous, pilose petiole and surface of second leaf, L/W ratio of second leaf up to 0.91.9 .
The second cluster split off at distance level 1.4 into two subclusters, the first subcluster split off into two groups at distance level 1.34, the first group represented by $T$. squarrosum and characterized by acuminate radicle apex, radicle length 2.3-2.5 $\mathrm{mm}$, cotyledonary blade $\mathrm{L} / \mathrm{W}$ ratio 3 , obovate; first leaf obovate; the second leaf oblongobcordate.

The second group represented by $T$. lappaceum and characterized by acute radicle apex, radicle length 1-1.2 mm, cotyledonary blade $\mathrm{L} / \mathrm{W}$ ratio 1.6 , first leaf oblate, the second leaf obcordate.

The second subcluster split off at distance level 1.38 into two groups, the first group represented by $T$. physodes and characterized by brown cotyledon colour, radicle position accumbent, petiole length of first leaf 28-29 $\mathrm{mm}$, dentate (upper 1/3) margin, $\mathrm{L} / \mathrm{W}$ ratio of second leaf 1.4.

The second group characterized by pale brown, pale yellow, radicle position incumbent, first leaf with petiole length up to $27 \mathrm{~mm}$; entire, dentate, serrate margin, the second leaf $\mathrm{L} / \mathrm{W}$ ratio up to 1.3 . 
The second group split off at 1.35 into two subgroups, the first subgroup represented by $T$. michelianum and characterized by cotyledonary ratio 4.5 , unifoliate blade shape obcordate with truncated apex and serrate margin.

The second subgroup characterized by cotyledonary ratio up to 2.7 , unifoliate blade shape square, depressed ovate, oblate, broadly ovate, ovate, cordate with acute, emarginate apex and entire, dentate margin.

The second subgroup split off at 1.3 into two classes, the first class represented by T. fragiferum and characterized by subacute radicle apex, hypocotyl green, first leaf with pubescent petiole surface; petiole length of second leaf 25-26 mm.

The second class characterized by acuterounded radicle apex, hypocotyl pale greenyellowish green, first leaf with glabrous-pilose petiole surface and petiole length of second leaf up to $22 \mathrm{~mm}$.

The second class split off at distance level 1.27 into two subclass, the first subclass split of at distance level 1.17 into two clades. The first clade represented by T. tomentosum and characterized by elliptic cotyledon shape, rounded radicle apex, mucilage attached to embryo, oblong cotyledon shape, petiole length of first leaf $23-27 \mathrm{~mm}$; ovate with acute apex, petiole length of second leaf $28-30 \mathrm{~mm}$; elliptic with acuminate apex.

The second clade represented by $T$. glomeratum and characterized by obovate cotyledon shape, acute radicle apex, mucilage non-attached to embryo, obovate cotyledon shape, petiole length of first leaf up to $15 \mathrm{~mm}$; broadly ovate with emarginated-apiculate apex, petiole length of second leaf $20-22 \mathrm{~mm}$; obcordate with emarginate apex.

The second subclass split off at distance level 1.24 into two clades, the first clade represented by $T$. repens, and characterized by cotyledon $\mathrm{L} / \mathrm{W}$ ratio up to 1.6 , yellowish green hypocotyl colour, obtuse cotyledon apex, cordate unifoliate leaf with dentate (upper 1/2) margin, the second leaf with serrate margin.
The second clade characterized by cotyledon L/W ratio 1.9-2.7, pale green hypocotyl colour, rounded cotyledon apex, square, depressed ovate, oblate, broadly ovate first leaf with entire margin, the second leaf with entire margin.

The second clade split off at distance 1.16 into two subclades, the first subclade represented by $T$. nigrescens and characterized by obovate cotyledon shape, obtuse radicle apex, first leaf broadly ovate with dentate margin and reniform base, second leaf circular with rounded base.

The second subclade represented by $T$. bocconei, T. campestre, T. dubium and characterized by oblong, elliptic cotyledon shape, acute radicle apex, square, depressed ovate, oblate first leaf with entire margin and truncated base, obcordate, cordate second leaf shape with cuneate, truncated base.

The second subclade split off at distance 1.1 which represented by $T$. bocconei characterized by radicle length up to $0.5 \mathrm{~mm}$, mucilage attached to embryo, first leaf square, petiole and blade pilose; second leaf petiole and blade pilose.

T. campestre and T. dubium separated at distance $1.03, T$. campestre characterized by elliptic cotyledon shape, hypocotyl length up to $12 \mathrm{~mm}$, first leaf depressed ovate, petiole length up to $6 \mathrm{~mm}$, second leaf cordate with cuneate base, petiole length up to $6 \mathrm{~mm}$.

T. dubium characterized by oblong cotyledon shape, hypocotyl length 17-19 mm, first leaf oblate, petiole length $9-10 \mathrm{~mm}$, second leaf obcordate with truncated base, petiole length $11-12 \mathrm{~mm}$.

\section{Discussion}

The important of seedling morphological characters such as cotyledons shape and colour, radicle shape, colour and position and plumule colour, prophyl leaf shape and margin were variable and could be used to identify Trigonella species (Abozeid et al., 2017) and Vicia species (Abozeid et al., 2018). 
In present study, Trifolium species possess many characters that play an important role in the differentiation between the studied species; cotyledon leaf shape; radicle apex, second leaf shape distinguished T. tomentosum from other species; Cotyledon colour, radicle apex, first leaf shape, second leaf hairness (indumentums) distinguished $T$. campestre from $T$. ligusticum; radicle apex, cotyledon $\mathrm{L} / \mathrm{W}$ ratio, hypocotyl length, first and second leaf shape distinguished $T$. physodes from $T$. michelium. Radicle apex, first leaf base, second leaf shape distinguished $T$. nigrescens from other species.

Cotyledon L/W ratio, mucilage attachment to embryo, cotyledon leaf shape, first leaf shape, second leaf margin distinguished $T$. glomeratum from $T$. repens.

Radicle apex, hypocotyls colour, first and second leaf shape distinguished $T$. argutum from, $T$. fragiferum. Radicle apex, cotyledon leaf shape distinguished $T$. squarosum from other species. Mucilage attachment to embryo, cotyledon L/W ratio, first leaf hairness (indumentums), second leaf base distinguished T. dubium from other species.

Cotyledon L/W ratio, first leaf shape distinguished $T$. bocconei from other species. Radicle shape, hypocotyls colour, first and second leaf shape distinguished $T$. lappaceum from T. purpureum. Sinjushin and Akopian (2011) considered seedling features of Vavilovia, Pisum and Lathyrus can used to identify species of studied genera. Feitoza $e t$ al. (2014) considered variation in embryos, plumule characters of Macrolobium Schreb. species (Leguminosae, Caesalpinioideae) provided characters with taxonomic relevance that differing among the studied species and aid species identification.

In conclusion, it is clear that embryo, cotyledonary, seedling, and first foliage leaf features are the most useful characters in the identification of Trifolium species as shown in the following key:

1a. Cotyledon shape elliptic........................................................

1b. Cotyledon shape oblong, obovate............................................. 4

2a. Radicle apex rounded, cotyledon leaf base cuneate, first leaf apex acute, second leaf elliptic. T. tomentosum

2b. Radicle apex acute, obtuse, cotyledon leaf base rounded, first leaf apex emarginate, second leaf obcordate.............................................

3a. Cotyledon pale yellow, radicle apex acute, first leaf depressed ovate, second leaf glabrous.....................................................T. campestre

3b. Cotyledon pale brown, radicle apex obtuse, first leaf oblate, second leaf pubescent......................................................... ligusticum

4a. Cotyledons obovate......................................................... 5

4b. Cotyledons oblong........................................................... 9

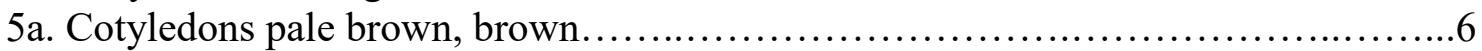

5b. Cotyledons pale yellow.................................................... 7

6a. Radicle apex obtuse, cotyledon L/W ratio up to1.9; hypocotyl length 18-19 $\mathrm{mm}$; first leaf ovate, second leaf elliptic............................. physodes

6b. Radicle apex acute, cotyledon $\mathrm{L} / \mathrm{W}$ ratio 4.5 ; hypocotyl length up to $8 \mathrm{~mm}$; first leaf obcordate, second leaf obcordate.........................T. michelianum

7a. Radicle apex obtuse, second leaf circular ........................ nigrescens

7b. Radicle apex acute, second leaf obcordate...................................... 8

8a. Cotyledon L/W ratio 2.3; mucilage not attached to embryo; cotyledonary leaf obovate, first leaf broadly ovate, the second leaf with dentate margin T. glomeratum

8b. Cotyledon L/W ratio 1.6; mucilage attached to embryo; cotyledonary leaf 
oblong, first leaf cordate, the second leaf with serrate margin...............T. repens

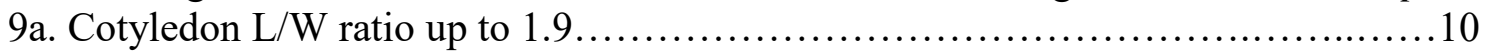

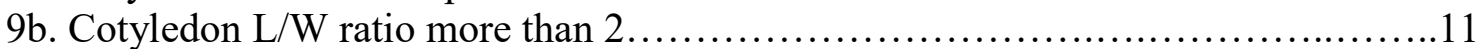

10a. Radicle apex acuminate, hypocotyl whitish green; first leaf oblong, second

leaf semicircular-obovate........................................ argutum

10b. Radicle apex acute, hypocotyl green, first leaf broadly ovate, second leaf elliptic...................................................... fragiferum

11a. Radicle apex acuminate; cotyledonary leaf obovate.....................

11b. Radicle apex acute, cotyledonary leaf oblong................................ 12

12a. First unifoliate leaf glabrous with truncated base........................... . dubium

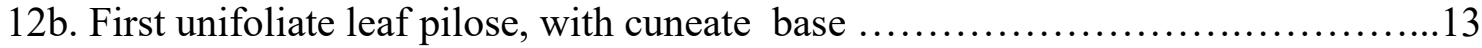

13a. First unifoliate leaf square.............................................. bocconei

13b. First unifoliate leaf oblate, elliptic.......................................... 14

14a. Radicle cylindrical; hypocotyl reddish green; first unifoliate leaf oblate, second leaf obcordate. T. lappaceum

14b. Radicle semicylindrical; hypocotyl pale green, first unifoliate leaf elliptic, second leaf obovate T. purpureum

\section{Acknowledgment}

My grateful thanks to Prof. Dr. Zaki A. Turki Professor of Plant Taxonomy and Flora, Botany and Microbiology Department, Faculty of Science, Menoufia University for his revision and helpful comments that improved the manuscript.

\section{Conflict of interest}

The authors declare that there are no conflicts of interest.

\section{References}

Abozeid, A., Turki, Z., El-Shayeb, F. \& Tang, Z. 2017. Embryo and seedling morphology of some Trigonella L. species (Fabaceae) and their taxonomic importance. Flora, 230: 57-65.

Abozeid, A., Liu, Y., Liu, J. \& Tang, Z. 2018. Taxonomic implication of embryo micromorphology in the genus Vicia L. (Fabaceae). Plant Syst. Evol., 304:33-42.

Ahammed, J. \& Paria, N., 1996. Systematic value of seedling morphology in some Indian Asteraceae. Acta Bot. Indica. 24 (1): 49-55.

Allen, O.N. \& Allen, E.K. 1981. The Leguminosae. A Source Book of Characteristics, Uses and Nodulation. Madison, University of Wisconsin Press. 812 pp.

Bandyopadhyay, S. 2002. Seedling morphology of Bauhinia foveolata Dalz.,
Leguminosae: Caesalpinioideae, Bombay Natural history Society. 99(3): 551-553.

Barbosa, C.Z., de Mendonça, M.S. \& Rodrigues, R.S. 2014. Seedling morphology of three sympatric savanna species of Byrsonima: first evidence of cryptogeal germination in Malpighiaceae and an overlooked seedling type in eudicots. Flora. 209 (8): 401-407.

Baudet, J.C. 1974. Signification taxonomique des caractères blastogéniquesdans la tribu des papilionaceae-phaseoleae. Bull. Jard. Bot. Nat. Belg. 44: 259-293.

Bokdam, J. 1977. Seedling morphology of some African Sapotaceae and its taxonomical significance. Meded. Landbouwhogeschool, Wageningen, the Netherlands. 77: 1-84.

Buendía Lázaro, F., Ruiz del Castillo, J. \& Tella Ferreiro, G. 1966. Semillas y plántulas de leguminosas pratenses españolas. Ministerio de Agricultura, Direccion General de Montes, Caza y Pesca Fluvial, Instituto Forestal de Investigaciones y Experiencias. 248 pp.

Burger, H.D. 1972. Seedling of some tropical trees and shrubs, mainly of South-East Asia. Centre for Agricultural publishing and Documentation (Pudoc), Wageningen. 399 pp. Compton, R.H. 1912. An investigation of the seedling structure in the Leguminosae. Bot. J. Linn. Soc. 41: 1-122. 
Conde, L.F. \& Stone, D.E. 1970. Seedling morphology in the Juglandaceae. The cotyledonary node. J. Arnold Arbor. 51: 463477.

Das, S. 2001. Seedling morphology of three mangrove species and its taxonomic implications. J. Plant Biol. 44(2): 92-96.

Duke, J.A. \& Polhill, R.M. 1981. Seedlings of leguminosae. in Polhill, R.M. and P.H. Raven (eds.). Advances in Legume systematics. Vol. II, Royal Botanic Gardens, Kew. pp 941-949.

Ellison, N.W., Liston, A., Stein er, J.J., Williams, W.M. \& Taylor, N.L. 2006. Molecular phylogenetics of the clover genus (Trifolium-Leguminosae). Mol. Phyl. Evol. 39: 688-705.

Farnsworth, E. J. 2008. Physiological and morphological changes during early seedling growth: roles of phytohormones. In: M.A. Leek, v.t. Parker, R.L. Simpson, (Eds), Seedling Ecology and Evolution. Cambridge University press, Cambridge, UK, pp. 150171.

Feitoza, G.V., dos Santos, J.U. M., Gurgel, E.S.C. \& Oliveira, D.M.T. 2014. Morphology of fruits, seeds, seedlings and saplings of three species of Macrolobium Schreb. (Leguminosae, Caesalpinioideae) in the Brazilian Amazon floodplain. Acta Bot. Bras. 28(3): 422-433.

Gavadi, F. \& Yamaguchi, H. 2004. A note on seed coat and plumule morphological variation in the genus Cicer L. (Fabaceae). Sci. Rep. Grad. Sch. Agric. \& Biol. Sci. Osaka Pref. Univ. 56: 7-16.

Hickey, L.J. 1973. Classification of the architecture of dicotyledonous leaves. Am. J. Bot. 60(1): 17-33.

Karaismailoglu, M.C. 2015. Morphological and anatomical features of seeds of Turkish Romulea taxa (Iridaceae) and their taxonomic significance. Acta. Bot. Croat.74: 31-41.

Kendir, G., Güvenç, A., Acar, A., Çeter, T. \& Pinar, N.M. 2015. Fruits, seeds and pollen morphology of Turkish Ribes L. (Grossulariaceae). Pl. Syst. Evol. 301: 185199.

Khalik, K.A. \&Van der Maesen, L. 2002. Seed morphology of some tribes of
Brassicaceae (implications for taxonomy and species identification for the flora of Egypt). Blumea. 47(2): 363-383.

Khalik, K.A., Van der Maesen, L., Koopman, W. \& Van den Berg, R. 2002. Numerical taxonomic study of some tribes of Brassicaceae from Egypt. Pl. Syst. Evol. 233: 207-221.

Lima, H.D. 1989. Tribo Dalbergieae (Leguminosae: Papilionoideae): morfologia dos frutos, sementes e plantulas e suaaplicacaona sistematica. Arq. Jard .Bot. Rio de Janeiro. 30: 1-42.

Lubbock, J. 1892. A contribution to our knowledge of Seedlings. Vols. 1\&2. Kegan Paul, Trench, Trübner, \& Co. LTD., London. 646 pp.

Muller, F. M. 1978. Seedling of the NorthWestern European lowland: A flora of seedlings, Centre for Agricultural publishing and documentation (Pudoc), Wageningen, 654 pp.

Mundhra, A., Roy, B. \& Paria, N.D. 2012. Investigation on Seedling Morphology in Taxonomic Studies of Angiosperms. In: Maiti, G., and Mukherjee, S.K. (eds), Multidisciplinary approaches in Angiosperm Systematics; University of Kalyani, Kalyani, West Bengal. pp. 64-72.

Nemoto, T., \& Ohashi, H. 1993. Seedling morphology of Lespedeza (Leguminosae).J. Pl. Res. 106:121-128.

Nozzolillo, C. 1985. Seedling morphology and anatomy of eight Cicer species and their taxonomic value. Can. J. Bot. 63(1): 1-6.

Oliveira, D.M.T. 2001. Morfologia comparada de plântulas e plantas jovens de leguminosa sarbórea snativas: espécies de phaseoleae, sophoreae, swartzieae e tephrosieae. Rev. Bras. Bot. 24 (1): 85-97.

Paria, N., Bhattacharyya, B. \& Ghosh, M. 1991. Seedling morphology of some members of Malvales with a note on their cytology. $J$. Nat. Bot. Soc. 44: 1-11.

Rodrigues, R.S. \& de Azevedo Tozzi, A.M.G. 2007. Morphological analysis and reexamination of the taxonomic circumscription of Acosmium (Leguminosae, Papilionoideae, Sophoreae). Taxon. 56: 439-452. 
Rohlf, F.J. 2000. NTSYS-pc: numerical taxonomy (Vitis vinifera L.) varieties usingmorphological data and multivariate analysis system. Exeter software and AFLP markers. Electr. J. Biotechnol. 6: 37-45.

Sanyal, S. \& Paria, N.D. 2015. Seedling morphology as a tool for taxonomic study in some members of Leguminosae (Fabaceae). Int. J. Plant Anim. Environ. Sci. 5 (1): 1-15.

Scott, R.C. \& Smith, D.L. 1998. Cotyledon architecture and anatomy in the Acacieae (Leguminosae: Mimosoideae). Bot. J. Linn. Soc. 128:15-44.

Sinjushin, A.A. \& Akopian, J.A. 2011. On seedling structure in Pisum L., Lathyrus L. and Vavilovia Fed. (Fabeae: Fabaceae). Wulfenia 18: 81-93.

Smith, D.L. 1983. Cotyledon anatomy in the Leguminosae. Bot. J. Linn. Soc. 86:325-355.

Tillich, H.J. 2003. Seedling morphology in Iridaceae: Indications for relationships within the family and to related families. Flora.198: 220-242.

Turki, Z., El-Shayeb, F. \& Abozeid, A. 2013.
Seed morphology of some Trigonella L. species (Fabaceae) and its taxonomic significance. Int. J. Sci. Res. 3: 940-948.

Turki, Z.A., Hegazy, M.M., El-Shayeb, F.M. \& El-Lithy, M.E. 2016. Seedling morphology of some Medicago species (Fabaceae) and its taxonomic implications. Egypt. J. Bot., Six International Conference. pp. 449-470.

Van de Wouw, M., Maxted, N., Chabane, K., \& Ford-Lloyd B.V. 2001. Molecular taxonomy of Vicia ser. Vicia based on amplifiedfragment length polymorphisms. $\mathrm{Pl}$. Syst. Evol. 229: 91-105.

Vogel, E.F. de. 1980. Seedlings of dicotyledons. Centre for Agricultural Publication and Documentation (Pudoc), Wageningen. $465 \mathrm{pp}$.

Ye, N. 1983. Studies on the seedling types of dicotyledonous plants (Magnoliophyta, Magnoliopsida). Phytologia. 54: 161-189.

Zohary, M. \& Heller, D. 1984. The genus Trifolium. Israel Academy of Science and Humanities. Jerusalem, Israel. 605pp. 
Appendix 1. Data matrix of $(0,1)$ codes of different characters states used in statistical analysis of Trifolium L. species (1) T. argutum, (2) T. bocconei, (3) T. campestre, (4) T. dubium, (5) T. fragiferum, (6) T. glomeratum, (7) $T$. lappaceum, (8) T. ligusticum, (9) T. michelianum, (10) T. nigrescens, (11) T. physodes, (12) T. purpureum, (13) $T$. repens, (14) $T$. squarrosum, (15) $T$. tomentosums

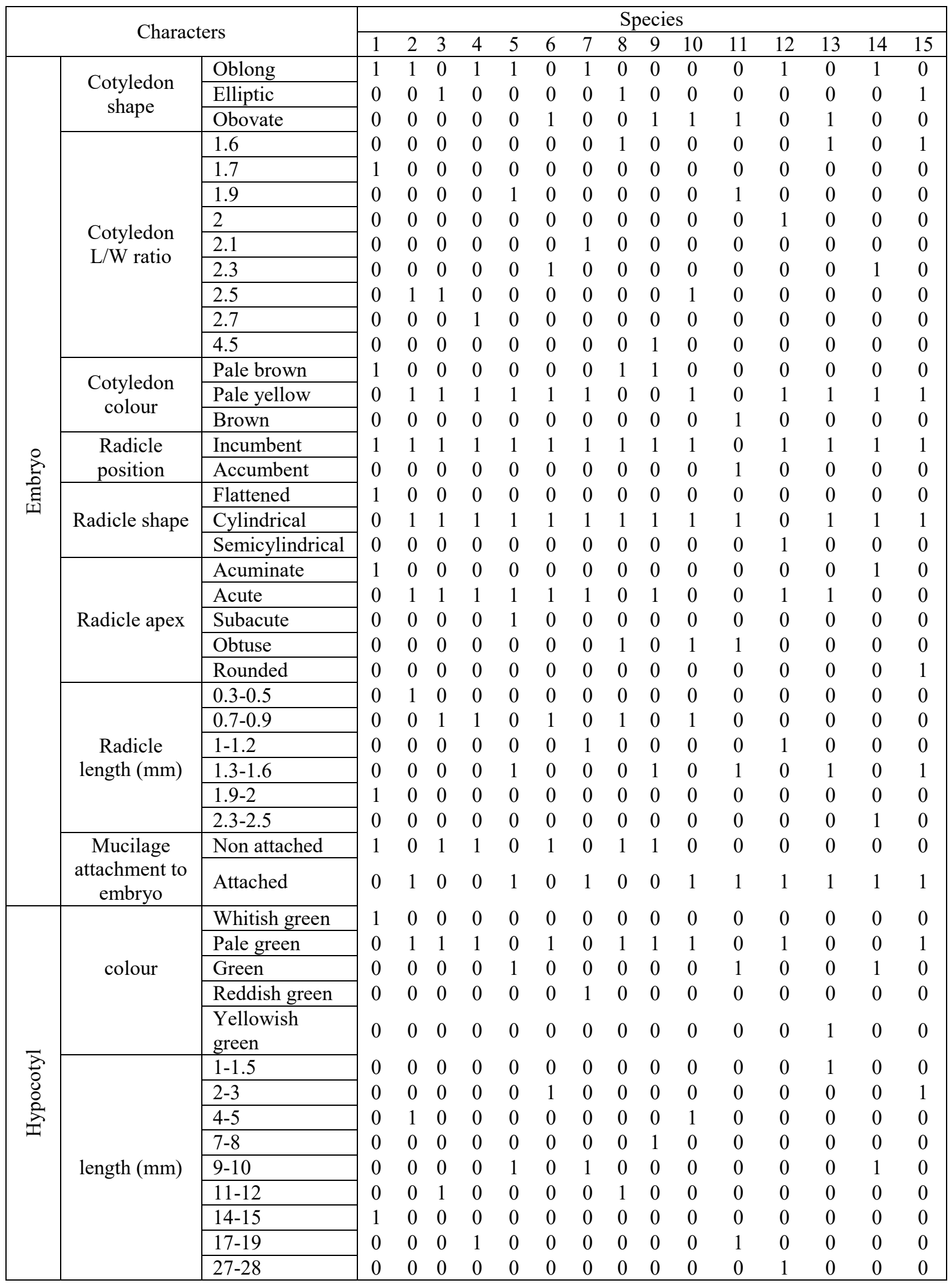


Embryo and seedling morphology of some Trifolium L. species

\begin{tabular}{|c|c|c|c|c|c|c|c|c|c|c|c|c|c|c|c|c|c|}
\hline \multirow{17}{*}{ 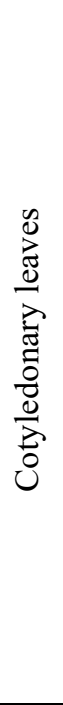 } & \multirow{2}{*}{ Blade shape } & Oblong & 1 & 1 & 1 & 1 & 1 & 0 & 1 & 1 & 1 & 1 & 1 & 1 & 1 & 0 & 1 \\
\hline & & Obovate & 0 & 0 & 0 & 0 & 0 & 1 & 0 & 0 & 0 & 0 & 0 & 0 & 0 & 1 & 0 \\
\hline & \multirow{2}{*}{ Blade apex } & Rounded & 1 & 1 & 1 & 1 & 1 & 1 & 1 & 0 & 1 & 1 & 1 & 1 & 0 & 1 & 1 \\
\hline & & Obtuse & 0 & 0 & 0 & 0 & 0 & 0 & 0 & 1 & 0 & 0 & 0 & 0 & 1 & 0 & 0 \\
\hline & \multirow{2}{*}{ Blade base } & Rounded & 1 & 1 & 1 & 1 & 1 & 0 & 1 & 1 & 1 & 1 & 1 & 1 & 1 & 1 & 0 \\
\hline & & Cuneate & 0 & 0 & 0 & 0 & 0 & 1 & 0 & 0 & 0 & 0 & 0 & 0 & 0 & 0 & 1 \\
\hline & \multirow{8}{*}{$\begin{array}{l}\text { Blade } \mathrm{L} / \mathrm{W} \\
\text { ratio }\end{array}$} & 1.3 & 0 & 0 & 0 & 0 & 0 & 0 & 0 & 0 & 0 & 1 & 0 & 0 & 0 & 0 & 0 \\
\hline & & 1.5 & 0 & 1 & 1 & 0 & 1 & 0 & 0 & 0 & 0 & 0 & 0 & 1 & 0 & 0 & 0 \\
\hline & & 1.6 & 0 & 0 & 0 & 0 & 0 & 0 & 1 & 0 & 1 & 0 & 1 & 0 & 0 & 0 & 0 \\
\hline & & 1.8 & 0 & 0 & 0 & 0 & 0 & 0 & 0 & 1 & 0 & 0 & 0 & 0 & 0 & 0 & 0 \\
\hline & & 1.9 & 0 & 0 & 0 & 0 & 0 & 1 & 0 & 0 & 0 & 0 & 0 & 0 & 1 & 0 & 0 \\
\hline & & 2 & 1 & 0 & 0 & 0 & 0 & 0 & 0 & 0 & 0 & 0 & 0 & 0 & 0 & 0 & 0 \\
\hline & & 2.1 & 0 & 0 & 0 & 1 & 0 & 0 & 0 & 0 & 0 & 0 & 0 & 0 & 0 & 0 & 1 \\
\hline & & 3 & 0 & 0 & 0 & 0 & 0 & 0 & 0 & 0 & 0 & 0 & 0 & 0 & 0 & 1 & 0 \\
\hline & \multirow{3}{*}{$\begin{array}{l}\text { Petiole length } \\
\quad(\mathrm{mm})\end{array}$} & $1-2$ & 0 & 1 & 1 & 1 & 0 & 0 & 0 & 1 & 0 & 1 & 0 & 0 & 1 & 0 & 0 \\
\hline & & $2.1-3$ & 0 & 0 & 0 & 0 & 0 & 1 & 1 & 0 & 0 & 0 & 0 & 1 & 0 & 1 & 1 \\
\hline & & $5-7$ & 1 & 0 & 0 & 0 & 1 & 0 & 0 & 0 & 1 & 0 & 1 & 0 & 0 & 0 & 0 \\
\hline \multirow{40}{*}{ 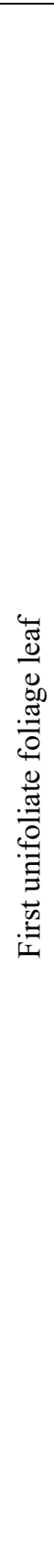 } & \multirow{10}{*}{$\begin{array}{l}\text { Petiole length } \\
\quad(\mathrm{mm})\end{array}$} & $3-4$ & 0 & 0 & 0 & 0 & 0 & 0 & 1 & 0 & 0 & 0 & 0 & 0 & 0 & 0 & 0 \\
\hline & & $5-6$ & 0 & 0 & 1 & 0 & 0 & 0 & 0 & 0 & 0 & 0 & 0 & 0 & 0 & 1 & 0 \\
\hline & & $7-8$ & 0 & 1 & 0 & 0 & 0 & 0 & 0 & 0 & 0 & 0 & 0 & 0 & 1 & 0 & 0 \\
\hline & & $9-10$ & 0 & 0 & 0 & 1 & 0 & 0 & 0 & 0 & 0 & 0 & 0 & 0 & 0 & 0 & 0 \\
\hline & & $12-15$ & 0 & 0 & 0 & 0 & 0 & 1 & 0 & 0 & 0 & 1 & 0 & 0 & 0 & 0 & 0 \\
\hline & & $17-20$ & 0 & 0 & 0 & 0 & 1 & 0 & 0 & 1 & 0 & 0 & 0 & 0 & 0 & 0 & 0 \\
\hline & & $23-27$ & 0 & 0 & 0 & 0 & 0 & 0 & 0 & 0 & 1 & 0 & 0 & 0 & 0 & 0 & 1 \\
\hline & & $28-29$ & 0 & 0 & 0 & 0 & 0 & 0 & 0 & 0 & 0 & 0 & 1 & 0 & 0 & 0 & 0 \\
\hline & & $30-31$ & 1 & 0 & 0 & 0 & 0 & 0 & 0 & 0 & 0 & 0 & 0 & 0 & 0 & 0 & 0 \\
\hline & & $39-41$ & 0 & 0 & 0 & 0 & 0 & 0 & 0 & 0 & 0 & 0 & 0 & 1 & 0 & 0 & 0 \\
\hline & \multirow{3}{*}{$\begin{array}{l}\text { Petiole } \\
\text { surface }\end{array}$} & Glabrous & 1 & 0 & 1 & 1 & 0 & 1 & 0 & 0 & 1 & 1 & 1 & 0 & 1 & 0 & 1 \\
\hline & & Pilose & 0 & 1 & 0 & 0 & 0 & 0 & 1 & 0 & 0 & 0 & 0 & 1 & 0 & 1 & 0 \\
\hline & & Pubescent & 0 & 0 & 0 & 0 & 1 & 0 & 0 & 1 & 0 & 0 & 0 & 0 & 0 & 0 & 0 \\
\hline & \multirow{10}{*}{ Blade shape } & Oblong & 1 & 0 & 0 & 0 & 0 & 0 & 0 & 0 & 0 & 0 & 0 & 0 & 0 & 0 & 0 \\
\hline & & Square & 0 & 1 & 0 & 0 & 0 & 0 & 0 & 0 & 0 & 0 & 0 & 0 & 0 & 0 & 0 \\
\hline & & depressed ovate & 0 & 0 & 1 & 0 & 0 & 0 & 0 & 0 & 0 & 0 & 0 & 0 & 0 & 0 & 0 \\
\hline & & Oblate & 0 & 0 & 0 & 1 & 0 & 0 & 1 & 1 & 0 & 0 & 0 & 0 & 0 & 0 & 0 \\
\hline & & broadly ovate & 0 & 0 & 0 & 0 & 1 & 1 & 0 & 0 & 0 & 1 & 0 & 0 & 0 & 0 & 0 \\
\hline & & Obcordate & 0 & 0 & 0 & 0 & 0 & 0 & 0 & 0 & 1 & 0 & 0 & 0 & 0 & 0 & 0 \\
\hline & & Ovate & 0 & 0 & 0 & 0 & 0 & 0 & 0 & 0 & 0 & 0 & 1 & 0 & 0 & 0 & 1 \\
\hline & & Elliptic & 0 & 0 & 0 & 0 & 0 & 0 & 0 & 0 & 0 & 0 & 0 & 1 & 0 & 0 & 0 \\
\hline & & Cordate & 0 & 0 & 0 & 0 & 0 & 0 & 0 & 0 & 0 & 0 & 0 & 0 & 1 & 0 & 0 \\
\hline & & Obovate & 0 & 0 & 0 & 0 & 0 & 0 & 0 & 0 & 0 & 0 & 0 & 0 & 0 & 1 & 0 \\
\hline & \multirow{6}{*}{$\begin{array}{l}\text { Blade } \mathrm{L} / \mathrm{W} \\
\text { ratio }\end{array}$} & 0.8 & 0 & 0 & 1 & 1 & 0 & 0 & 1 & 1 & 1 & 1 & 0 & 0 & 0 & 0 & 0 \\
\hline & & 0.9 & 0 & 0 & 0 & 0 & 1 & 0 & 0 & 0 & 0 & 0 & 0 & 0 & 0 & 0 & 0 \\
\hline & & 1 & 0 & 1 & 0 & 0 & 0 & 0 & 0 & 0 & 0 & 0 & 0 & 0 & 1 & 0 & 1 \\
\hline & & 1.1 & 0 & 0 & 0 & 0 & 0 & 1 & 0 & 0 & 0 & 0 & 1 & 0 & 0 & 1 & 0 \\
\hline & & 1.3 & 1 & 0 & 0 & 0 & 0 & 0 & 0 & 0 & 0 & 0 & 0 & 0 & 0 & 0 & 0 \\
\hline & & 2.1 & 0 & 0 & 0 & 0 & 0 & 0 & 0 & 0 & 0 & 0 & 0 & 1 & 0 & 0 & 0 \\
\hline & \multirow{5}{*}{ Blade apex } & Acute & 1 & 0 & 0 & 0 & 0 & 0 & 0 & 0 & 0 & 0 & 0 & 0 & 0 & 0 & 1 \\
\hline & & Emarginate & 0 & 1 & 1 & 1 & 1 & 0 & 0 & 1 & 0 & 1 & 1 & 1 & 1 & 1 & 0 \\
\hline & & $\begin{array}{l}\text { Emargainate } \\
\text { apiculate }\end{array}$ & 0 & 0 & 0 & 0 & 0 & 1 & 0 & 0 & 0 & 0 & 0 & 0 & 0 & 0 & 0 \\
\hline & & Rounded & 0 & 0 & 0 & 0 & 0 & 0 & 1 & 0 & 0 & 0 & 0 & 0 & 0 & 0 & 0 \\
\hline & & Truncate & 0 & 0 & 0 & 0 & 0 & 0 & 0 & 0 & 1 & 0 & 0 & 0 & 0 & 0 & 0 \\
\hline & & Rounded & 1 & 0 & 0 & 0 & 0 & 0 & 1 & 0 & 0 & 0 & 0 & 0 & 0 & 0 & 0 \\
\hline & Plade $\mathrm{b}$ & Truncate & 0 & 1 & 1 & 1 & 1 & 1 & 0 & 1 & 1 & 0 & 1 & 0 & 1 & 0 & 1 \\
\hline & Draue Dase & Reniform & 0 & 0 & 0 & 0 & 0 & 0 & 0 & 0 & 0 & 1 & 0 & 0 & 0 & 0 & 0 \\
\hline & & Cuneate & 0 & 0 & 0 & 0 & 0 & 0 & 0 & 0 & 0 & 0 & 0 & 1 & 0 & 1 & 0 \\
\hline & Pl & Ciliate & 1 & 0 & 0 & 0 & 0 & 0 & 0 & 0 & 0 & 0 & 0 & 0 & 0 & 0 & 0 \\
\hline & Blade marg1 & Entire & 0 & 1 & 1 & 1 & 0 & 0 & 1 & 1 & 0 & 0 & 0 & 1 & 0 & 1 & 0 \\
\hline
\end{tabular}




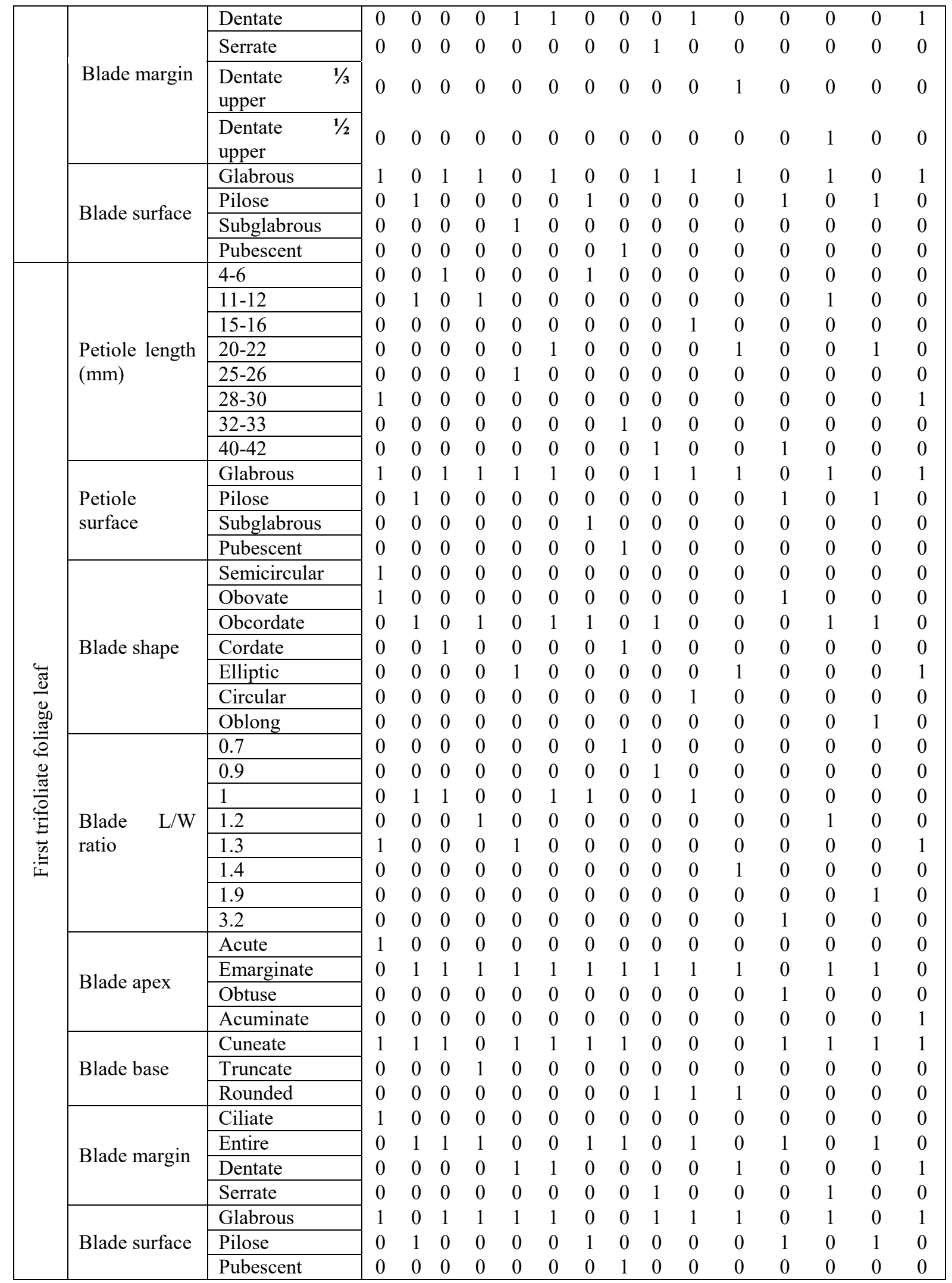

